Time-Variation of Higher Moments in a Financial Market with Heterogeneous Agents: An Analytical Approach

Simone Alfarano, Thomas Lux and Friedrich Wagner 


\title{
Time-Variation of Higher Moments in a Financial Market with Heterogeneous Agents: An Analytical Approach
}

\author{
Simone Alfarano, Thomas Lux and Friedrich Wagner
}

August 16, 2005

\begin{abstract}
A growing body of recent literature allows for heterogenous trading strategies and limited rationality of agents in behavioral models of financial markets. More and more, this literature has been concerned with the explanation of some of the stylized facts of financial markets. It now seems that some previously mysterious time-series characteristics like fat tails of returns and temporal dependence of volatility can be observed in many of these models as macroscopic patterns resulting from the interaction among different groups of speculative traders. However, most of the available evidence stems from simulation studies of relatively complicated models which do not allow for analytical solutions. In this paper, this line of research is supplemented by analytical solutions of a simple variant of the seminal herding model introduced by Kirman [1993]. Embedding the herding framework into a simple equilibrium asset pricing model, we are able to derive closed-form solutions for the time-variation of higher moments as well as related quantities of interest enabling us to spell out under what circumstances the model gives rise to realistic behavior of the resulting time series.
\end{abstract}

\section{Introduction}

Until very recently, theoretical research in finance has largely ignored some of the really universal stylized facts of practically all available financial data. In fact, a glance at frequently used textbooks like the ones by O'Hara [1995] and Barucci [2003] shows that even their glossaries lack entries for some of the prevalent technical terms of the empirical finance literature. For example, while many developments in empirical finance are essentially motivated by the observation of non-Gaussian returns distributions with their "fat tails" and temporal dependence of second moments leading to "volatility clustering", these notions have been almost entirely 
absent from the theoretical literature. ${ }^{1}$ While these phenomena have spurred the development of such seminal innovations like GARCH-type and stochastic volatility models in empirical finance, their behavioral origins have apparently remained an almost unaccessible puzzle for a long time. Two reasons might be responsible for this neglect: first, the above features characterize the behavior of financial time series as a whole, while the interest in economic theory has typically been to spell out the effect of a change of one (endogenous) economic variable on other, exogenous variables. Even when allowing for an ensemble of traders, such a comparative statics approach is not appropriate for explaining universal conditional and unconditional stochastic properties. Second, the prevalent efficient market paradigm did, in fact, provide a very simple implicit answer to the question of the origin of all stylized facts of returns: since, in this framework, prices would reflect forthcoming news in an unbiased and immediate manner, any property of the returns distribution would simply reflect a similar feature of the distribution of new information items. As a corollary of the efficient market hypothesis, the "news arrival process" would, therefore, have to come along with fat tails and clustering of important news. Unfortunately, this corollary can hardly be subjected to econometric scrutiny. On the other hand, enough evidence had been collected against the universal validity of the efficient market paradigm to motivate alternative, behavioral approaches which then mushroomed over the nineties.

First analyses of complex data generating mechanisms based on interacting agents can be found in Kirman [1991, 1993] and DeGrauwe et al. [1993]. While they did not focus then on the above stylized facts (not broadly acknowledged at that time among theoretical researchers), they both already showed that their models could mimic the random walk nature of asset prices and exchange rates although their data-generating processes were clearly different from a true random walk. Notably, both studies also investigated what might be described as secondary stylized facts: they applied certain frequent econometric analyses to their simulated data and found similar behavior as with empirical records providing a possible explanation of, e.g., the forward premium puzzle of foreign exchange markets.

Evidence for volatility clustering as an emergent phenomenon of a multi-agent model appeared first in Grannen and Swindle [1994]. While a large body of subsequent models studied artificial markets with heterogenous autonomous agents often endowed with some sort of artificial intelligence (classifier systems, genetic algorithms), any consideration of empirical stylized facts is also curiously absent in the first wave of such papers (Levy et al. [1994], Arifovic [1996], Arthur et al. [1997]). In fact, much of this early literature had been preoccupied with the question of conver-

\footnotetext{
${ }^{1}$ Perfectly the same holds for theoretical work on exchange rate determination where the same stylized facts have also remained unnoticed for a long time.
} 
gence or not of their learning algorithms to the benchmark of rational expectations rather than considering empirical applications. However, subsequent research has shown that relatively simple models of interacting traders could produce realistic time series sharing the "stylized facts" of fat tails and clustering volatility even up to numerical agreement with key quantities of empirical data (Lux and Marchesi $[1999,2000])$. Similar investigations of the dynamic properties of alternative models revealed that many agent-based approaches share a certain tendency of generating fat tails and volatility clustering although their quantitative manifestations are not always identical to the very robust numbers obtained with empirical data (cf. LeBaron et al. [1999], Chen and Yeh [2002], Kirman and Teyssière [2002], Lux and Schornstein [2005]). While often the general appearance of simulated data seemed to be quite robust with respect to most of the underlying parameters, it also turned out that the potential of generating stylized facts depends crucially on the system size (i.e., number of agents). Realistic dynamic patterns are typically observed with the (probably natural) initial choice of a few hundred or thousand agents. However with an increasing number of market participants one often experiences a fading away of the fat tails and volatility clustering beyond a certain threshold (cf. Egenter et al. [1999], Lux and Schornstein [2005], Challet and Marsili [2004]).

The present paper attempts to shed light on both of the findings detailed above. Within a relatively simple type of herding model (broadly along the lines of Kirman [1993]) we derive closed-form solutions for auto-covariances of returns and their higher moments together with other statistics such as mean-passage times. Inspection of the results allows to infer in how far and under what conditions the model could mimic the empirical findings of fat tails and clustering of volatility. Investigation of different specifications of the model also allows us to point out why in certain scenarios - increasing system size would lead to vanishing stylized facts. Our approach is broadly complementary to recent attempts at studying asymptotic properties of related agent-based models (Horst [2004], Föllmer et al. [2005]). These authors provide conditions under which the limiting distribution of the price process exists in models with both global and local interactions of agents (Horst [2004]) and models with feedback from the price process on the group dynamics (Föllmer et al. [2005]). Since our model can be viewed as a special case of the class of models studied in Horst [2004] his results on convergence of equilibrium prices to a unique equilibrium distribution also apply in our case. However, instead of focusing on the properties of the price process alone we extend the analysis to returns to which the famous empirical regularities of fat tails and clustered volatility apply. We also go beyond asymptotic convergence results by working out various properties of the stationary distribution which are of interest in the light of empirical findings.

The paper is organized as follows: section 2 introduces different variants of herding models inspired by Kirman's ant process [Kirman, 1993]. Section 3 incorporates 
the recruitment mechanism of the ant model into an asset pricing framework. Section 4 details our analytical results for both the unconditional and conditional distribution of returns. In section 5 we explain different techniques for simulating these agent-based models, while section 6 points out under which conditions the interesting dynamics survives for large numbers of agents. Section 7 concludes. Technical details are relegated to various appendices.

\section{The Ant Model and Its Financial Interpretation}

In his seminal 1993 paper, Kirman provides a simple stochastic formalization of information transmission inspired by macroscopic patterns emerging from entomologic experiments with ant colonies. The underlying scenario is one of foraging ants who have two identical sources of food at their disposal in the vicinity of their nest. Experimental settings show that, at any point in time, a majority of the ant population concentrates on exploiting one particular food source, but every once in a while a switch occurs from one to the other source. Thus, averaging over time, a bimodal distribution of the frequency of ants visiting one or the other manger would result. Kirman explains this phenomenon by a combination of pair-wise interaction (exchange of information by pheromons) and an autonomous switching probability due to stochastic search. With an increase of the strength of the herding component, the resulting stationary probability distribution of the model bifurcates from unimodality with an equal exploitation of both sources to a bi-modal one supporting the experimental findings.

In a long series of subsequent papers, this and similar mechanisms have been applied as formalizations of contagion effects in financial markets. Kirman [1991, 1993] replaces the binary choice of food sources by the ants by agents' choice of a particular rule for the formation of their expectations. In his adaption to foreign exchange market dynamics, agents can adopt chartist and fundamentalist forecasts of future exchange rate movements. With the herding mechanism governing agents' distri-

bution on these two behavioral types, the market switches between a dominance of fundamentalist and chartist behavior with periods in which the later prevails giving rise to speculative bubbles. Market equilibria are determined from a standard monetary exchange rate model in which the assumption of rational expectations of the original framework has been replaced by 'non-rational' expectations computed as the average of the expectations of the two groups of chartist and fundamentalist both weighted by a measure of their past success. Most relevant to our purpose, Kirman and Teyssière [2002] have analyzed the temporal dependence of returns and volatility from simulated data using a variety of pertinent econometric tools and have found similar indication for long-term dependence in second moments as with real-life data. 
A closely related variant of a herding model has been proposed by Lux [1995], who replaced the pair-interaction by an overall (mean-field) effect of the average influence of the whole ensemble of traders on each individual's choice of strategy. This setting allowed to derive a number of analytical results via application of the Master equation approach adopted from statistical physics (Lux [1997, 1998]). An extended version of this model (Lux and Marchesi [1999]) with switching between three different groups could be shown to give rise to realistic time series properties. Egenter et al. [1999] showed, however, that these results depended on the number of agents and that raising the number of market participants beyond several thousand individuals leads to a gradual fading away of all its 'interesting' dynamics. We will point out later what mechanisms are responsible for this loss of stylized facts and under which assumptions 'interesting' solutions can be preserved even in large markets.

To set the stage, let us first restate the mechanics of the herding model proposed by Kirman [1993]. The market (or ant colony) is populated by a fixed number of agents $N$, each of them being either in state 1 or 2 . The number of agents in the first state will be denoted by $n$, so that $n \in\{0,1, \ldots, N\}$ defines the configuration of the system (the number of agents in the opposite state will obviously be $N-n$ ). The stochastic evolution of the system is governed by random meetings of two agents, after which one of them might follow the companion, and autonomous switches, i.e. independent random changes of state. In the original set-up, the encounters of agents are formalized by pair-wise interaction involving two ants/agents at a time, which implies that the density of ants in the given search area is low in order to exclude the possibility of multiple meetings. Additionally, neither the probability of following another companion nor the success in recruiting companions depend on the outcome of previous meetings. The lack of memory of the agents is the crucial assumption to formalize the population dynamics as a Markov process. It, then, evolves according to the probabilities of changing from state $n$ at time $t$ to some state $n^{\prime}$ at time $t+\Delta t_{0}$. Let these Markovian conditional probabilities be denoted by $\rho\left(n^{\prime}, t+\Delta t_{0} \mid n, t\right)$ which are related to the transition rates per unit time, $\pi\left(n \rightarrow n^{\prime}\right)$ by $\rho\left(n^{\prime}, t+\Delta t_{0} \mid n, t\right)=\pi\left(n \rightarrow n^{\prime}\right) \Delta t_{0}$ for small time increments $\Delta t_{0}$. Since in the limit of continuous time $\Delta t_{0} \rightarrow 0$, multiple switches during one incremental time unit become increasingly unlikely, one can confine the analysis to $n^{\prime}=n \pm 1$ with transition rates:

$$
\begin{aligned}
& \pi(n \rightarrow n+1)=(N-n)\left(a+\bar{b} \frac{n}{N}\right), \\
& \pi(n \rightarrow n-1)=n\left(a+\bar{b} \frac{(N-n)}{N}\right) .
\end{aligned}
$$

The term proportional to the number of agents in the state models their random changes, while the quadratic term $\bar{b} \frac{(N-n) n}{N}$ governs the recruitment mechanism; the parameter $\bar{b}$ measures the strength of the influence of the companions ${ }^{2}$.

\footnotetext{
${ }^{2}$ In the original version $\bar{b}=1-\delta$, where $\delta$ is interpreted as the probability of an unsuccessful recruitment.
} 
Some comments are in order here. First, note that the transition rates are invariant under the transformation $n \rightarrow N-n$. It reflects the fact that the recruitment mechanism is independent of the type of agents, and the random switching component $a$ is equal in both states. The system, therefore, does not exhibit any preferential direction between the two states (in Alfarano et al. [2005] a more general asymmetric setting has been investigated). This underlying symmetry is responsible for the resulting symmetric shape of the unconditional distribution of ants' visiting time (see section 4.2). Moreover, the above transition rates are non-linear because of the presence of the quadratic dependence on $n$. In general, non-linearity in the transition rates arises from the presence of interactions among agents. In Kirman's model, an individual in state 1 is indirectly affected by agents who are in the other state thought the recruitment mechanism. Linear transition rates, conversely, describe uncorrelated movements between groups due to idiosyncratic motivations and, in general, systems composed by independent individuals.

An additional crucial property is the extensivity of the transitions in (1), since they satisfy the following relation:

$$
\pi\left(n \rightarrow n^{\prime}\right)=N \pi\left(z \rightarrow z^{\prime}\right)
$$

where $z=n / N$ stands for concentrations rather than the raw numbers of agents, $n$, as a variable to characterize the entire system. The extensivity property typically characterizes systems whose evolution is governed by interaction mechanisms involving a small number of neighbors -as compared to the population size. As we will see more precisely later on, this 'local' nature of the interactions among individuals has remarkable consequences in the 'thermodynamic' limit (i.e. when the number of individual $N$ becomes very large): the equilibrium probability distribution $\omega_{e}(x)$ of the concentration of agents $x$ becomes sharp around a macroscopic state variable, which might be defined by the mean ${ }^{3}$ of $\omega_{e}(x)$. In other words, the fluctuations of the system can be neglected, and its dynamics can be described by a deterministic differential equation (see sec. 6 below).

What happens if we relax the extensivity assumption (2) based on local interactions among individuals? In which way would the dynamics of the system change under the hypothesis of collective interactions? In order to study possible differences in these alternative scenarios, we introduce a new pair of transition rates, where the extensivity property (2) does not hold:

$$
\begin{aligned}
& \pi(n \rightarrow n+1)=(N-n)(a+b n), \\
& \pi(n \rightarrow n-1)=n(a+b(N-n)) .
\end{aligned}
$$

\footnotetext{
${ }^{3}$ Alternative definitions could be based on the mode, the median or other characterizations of the typical value of the distribution $\omega_{e}(x)$.
} 
The transition rates (3) describe a scenario where the interaction among agents does not depend on the fraction of agents in the alternative states, but rather on the overall number of such agents. More precisely, an individual might change state due to a random event, as in the previous setting, or he might switch under the group pressure of the overall number of the individuals that belong to the opposite state. A closer inspection to eqs. (1) and (3) shows that the main difference lies in the scaling properties of the herding parameter. In the first formalization we observe a dependence of the type $O(1 / N)$, with the herding parameter, vanishing in the limit $N \rightarrow \infty$. In the second setting, on the contrary, $b \sim O(1)$, which therefore remains bounded in this limit. One of the contributions of this paper is to point out the interesting consequences of this different formalization, and the implications in the characterization of the resulting dynamics in the two different scenarios, namely extensive versus non-extensive transition rates when we increase the number of individuals $N$.

Would it make intuitive sense to replace intensities by occupation numbers as we have done in eq. (3)? First, let us consider the autonomous switching component. Increasing the number of agents and keeping the Poisson probability $a$ of an autonomous switch by any particular agent per time unit constant, larger $n$ and $N-n$ would also lead to a higher probability of observing one such move within the time interval $\Delta t$. Since agents are independent in this autonomous component, the linearity in $n$ and $N-n$ assumed in eqs. (1) and (3), in fact, appears intuitively plausible. Replacing, for example, $n a$ by $\frac{n}{N} a$ in the second equation would amount to assuming that probability $a$ does not apply to each individual agent herself but rather to a fraction of the population. One can argue in the same way with respect to the herding component: increasing the overall number of ants or agents (and assuming that they are distributed equally in the space or have the same probability of meeting other agents), the likelihood for agents to randomly bump into each other should also increase, presumably linearly, in the number of agents (if we were not to incorporate particular topological considerations into the model). Vice versa, a formulation in intensities, i.e. expressing the conversion probabilities by $b \frac{n}{N}$ and $b \frac{N-n}{N}$ in eqs. (1) would amount to a constant probability of meeting agents from the other group independent of the overall number of agents which - at least in the literal interpretation of the model - would be implausible. If we imagine to increase the number of ants $N$ and to keep the area $S$ constant, the resulting increase of the density of the colony would lead to a higher frequency of pairwise contacts, and finally, to a uniform mass of ants covering all the area $S$. However, if we use the entomological information transmission mechanism as a parabola for herding among humans, we are not anymore constrained to a bounded region due to the 'non-spatial' nature of modern communication systems. It is, therefore, not obvious which of the two approaches would be preferable in an economic setting. Using eq. (1) to formalize the interactions, the extensivity property, then, constrains the information transmission 
to a limited number of neighbors. The alternative approach, formalized by eq. (3), describes a scenario with connectivity increasing with the number of agents. It can be used to model, for instance, groups pressure or mass-media interaction.

In the literature on agent-based economics, one often finds a formulation in which transition rates depend on intensities rather than on group occupation numbers, i.e. on $\frac{n}{N}$ and $\frac{N-n}{N}$ instead of $n$ and $N-n$ (Lux [1995]). Apparently, system size appears to become suppressed in such a formalization and one might argue that this would make the model outcome insensitive with respect to the market size (as it turns out, the opposite, in fact, happens). In order to investigate the effects of occupation number formalism vs. transition probabilities expressed in terms of concentrations later on, we can integrate this alternative approach into the framework of eqs. (3) by replacing the constant $b$ by a flexible form, $b=\frac{b_{0} N_{0}}{N}$ in which $b_{0}$ and $N_{0}$ are the benchmark values for some initial scenario with constant parameters $a$ and $b_{0}$ and benchmark population $N_{0}$. The changes brought about by an increasing population in the concentration formulation would, then, simply amount to multiplying the original herding constant by $\frac{N_{0}}{N}$ which obviously leads to an effective decrease of the succeptability of agents to contagion effects with increasing population size. For a sufficiently high value of $N$, this transformation triggers macroscopic changes in the underlying scenario, going from a bimodal equilibrium distribution to a 'trivial' Gaussian.

\section{The Financial Market}

In order to close the model, we embed the herding dynamics into a simple noise trader framework. In particular, we interpret group 1 and 2 as optimistic and pessimistic noise traders, respectively, who expect the prices to increase or decrease in the near future. Optimists are assumed to buy a certain number $T_{c}$ of additional units of the asset while pessimists sell $T_{c}$ units $\left(T_{c}\right.$, thus, is a measure of average transaction volume of chartists). Besides these chartists or noise traders, whose belief dynamics are described by the above herding model, the model also hosts a second group of fundamentalist traders who buy/sell if the current price $p$ is below/above their perceived fundamental value $p_{f}$. Assuming that this group has $N_{f}$ members with average trading volume $T_{f}$ and that they react on relative deviations between $p$ and $p_{f}$, excess demand by this group amounts to

$$
E D_{f}=N_{f} T_{f} \ln \left(\frac{p_{f}}{p}\right)
$$

Since noise traders' excess demand mainly derives from a dominance of optimism and pessimism in this sub-population, we can conveniently trace it back to a quantity describing the population configuration: $x=\frac{2 n}{N}-1$, which if zero signals a balanced 
disposition (zero excess demand) and if positive or negative amounts to optimistic or pessimistic majorities. Noise traders' excess demand, therefore, becomes $E D_{C}=$ $N T_{c} x$. Invoking a standard Walrasian price adjustment mechanism, relative price changes would depend on overall excess demand:

$$
\frac{1}{p} \frac{d p}{d t}=\beta\left(N_{f} T_{f} \ln \left(\frac{p_{f}}{p}\right)+N T_{c} x\right)
$$

with $\beta$ the assumed price adjustment speed. Except for the incorporation of the herding mechanism through $x$, this is a fairly standard asset price equation similar to what one finds in the legacy of models with chartist-fundamentalist interaction (e.g. Beja and Goldman [1980], Day and Huang [1990]).

Setting (without loss of generality) $N_{f} T_{f} / N T_{c}=1$ and assuming instantaneous market clearing $(\beta \rightarrow \infty)$, we arrive at the equilibrium price driven both by fundamental information and the average mood of noise traders:

$$
p(t)=p_{f} \exp (x(t)) .
$$

Hence, derivation of the limiting distribution of the configuration $x$ together with a specification of the fundamentals process would allow us to characterize the distribution of equilibrium prices. However, we are interested in returns rather than prices themselves as the later are known to be non-stationary in almost all real-life markets. From eq. (6), returns over arbitrary time horizons are computed as:

$$
r(t, \tau)=\ln p(t+\tau)-\ln p(t)=(x(t+\tau)-x(t)),
$$

assuming that $p_{f}$ is constant over time. Volatility can be obtained as $V(t, \tau)=$ $r^{2}(t, \tau)$. Eqs. (1) to (6) define a simple noise trader/infection framework with heterogenous interacting agents which is quite similar in spirit but simpler than the formalization of Kirman [1991], and Kirman and Teyssière [2002]. Since the population configuration from the infection process enters directly on the demand side of the model it is, in fact, possible to infer the statistical properties of the returns and volatility processes under the influence of this ant-like contagion dynamics.

It is interesting to compare the above framework to the way in which the ant model is incorporated into a monetary exchange rate model in Kirman [1991], Kirman and Teyssière [2002]. In their papers, the contagion dynamics occurs on a much faster time scale than price formation in the foreign exchange market. In particular, they extract the population configuration at integer times $t$ as a snapshot of the distribution of agents after a large number of pairwise meetings (for example, 10,000 meetings in Kirman [1991]) at the "intra-daily level". The current distribution is then used to compute the weights of chartists and fundamentalists and defining 
the market expectation as the average of current chartist and fundamentalist forecasts, and the equilibrium exchange rate is derived from a discrete-time monetary model. ${ }^{4}$ The separation of time scales allows to observe relatively large fluctuations of the average opinion at integer time steps, but it also impedes to take stock of the theoretical solution of the ant process for a formal analysis of the exchange rate dynamics. In contrast, in our approach, the time scales of the ant process and price formation are the same (although we will later distinguish between the continuous time dynamics of the process and discrete measurements thereof). With our Poisson probabilities, both occur in continuous time with changes of the configuration of noise traders coming along with changes in equilibrium prices.

\section{Analytical Results}

The structure of the model allows to apply the Master equation formalism in order to derive macroscopic properties of our dynamical system (cf. Aoki [2002]). Note that our financial market consists of a large collection of interacting jump processes with state-dependent and non-linear transition rates. In this paragraph we show how this formalism allows for a compact characterization of the aggregate dynamics of the system in terms of its underlying parameters.

\subsection{Fokker-Planck Approximation}

Sticking with our definition of non-extensive transition rates in eq. (3), the group dynamics is characterized by the transition probabilities for agents' moves between the two states. These transition probabilities define a Markovian stochastic process which belongs to the class of so-called nonlinear "one-step processes" (cf. Van Kampen [1992]). In the following, we approximate the Markovian process (3) by a continuous diffusion process governed by the Fokker-Planck equation. First note that the probabilities $\bar{\omega}(n, t)$ to have $n$ agents in state one at time $t$, obey the so-called Master equation, which gives the probability flux between states:

$$
\frac{\partial \bar{\omega}(n, t)}{\partial t}=\sum_{m}\left(\pi_{n, m} \bar{\omega}(m, t)-\pi_{m, n} \bar{\omega}(n, t)\right),
$$

with $\pi_{n, m}=\frac{\rho_{n, m}}{\Delta t_{0}}$ the transition rates from states $m$ to states $n$. In the limit $\Delta t_{0} \rightarrow 0$, multiple jumps occur with probability zero, so that one only has to consider jumps to neighboring states, i.e. $m=n \pm 1$. Replacing $n$ by the intensive variable $x$ and treating it, for large $N$, as a continuous quantity, we can derive a FokkerPlanck-equation for the time change of the pertinent probability density $\omega(x, t)$ (cf.

\footnotetext{
${ }^{4}$ There is, in fact, even a further intermediate step in which agents try to make an assessment of the prevailing majority opinion on the base of a noisy signal. The distribution of this observation rather than their own previous state is used in the computation of the weights of both groups.
} 
Appendix A.1 for details):

$$
\frac{\partial \omega(x, t)}{\partial t}=\frac{\partial}{\partial x}(A(x) \omega(x, t))+\frac{1}{2} \frac{\partial^{2}}{\partial x^{2}}(D(x) \omega(x, t)) .
$$

The drift and diffusion terms $A(x)$ and $D(x)$ in eq. (9) are respectively given by:

$$
A(x)=-2 a x, \quad D(x)=2 b\left(1-x^{2}\right)+\frac{4 a}{N} .
$$

The process is, therefore, characterized by a linear drift component with meanreversion towards its unconditional mean value $E[x]=0$. Additionally, the diffusion function, governing the properties of the fluctuations of the system, consists of two terms: one term is $\mathrm{N}$-dependent, and becomes negligible for large values of $N$; the second term is a symmetric and quadratic function of $x$, which is independent of $N$. The two terms characterize two different mechanisms for the emergence of endogenous fluctuations. The N-dependent term governs the fluctuations due to the intrinsic granularity of the system, which obviously has to vanish in the continuous approximation. The second term is directly related to the herding interactions among individuals. If we are not too close to the boundaries $x=1$ and $x=-1$, the herding mechanism dominates, and the fluctuations are governed by the quadratic term, while the $\mathrm{N}$-dependent term can be neglected ${ }^{5}$. However, near the boundaries, the two effects are comparable in magnitude, therefore the N-dependent term should also be taken into account. Moreover, it is a remarkable property of the system that the diffusion function does not depend on the number of agents $N$-if we neglect the granularity term. The magnitude of the fluctuations of the opinion index $x$ is, therefore, independent on the system size (i.e. in our case the number of agents), and we observe qualitatively similar behavior for systems with any number of agents (see Figure 1 in section 5). The 'collective' nature of interactions among individuals formalized due to the non-extensivity of the transition rates (3) is the ultimate reason for this particular behavior.

If we employ the original Kirman setting as the underlying scenario for the artificial market, the previous property of independence of the level of the fluctuations on the system size changes dramatically. Let us assume that the underlying transition rates between the two states are now governed by eq. (1) rather than by eq. (3). In this setting we can also derive a diffusion approximation for the dynamics of the stochastic process (1), pretty much in the same line as the derivation of the FPE (9) in the case of non-extensive transition rates (3). The main difference consists in replacing the parameter $b$ in the diffusion function (10) by $\frac{\bar{b}}{N}$. We, then, find the

\footnotetext{
${ }^{5}$ More precisely, the dominance of the herding term over the granular term is guaranteed if the inequality $2 b\left(1-x^{2}\right)>\frac{4 a}{N}$ holds, which is equivalent to constrain the variable $x$ to the interval $\left(\frac{2 a}{b N}-1,1-\frac{2 a}{b N}\right)$.
} 
following drift and diffusion functions:

$$
A(x)=-2 a x, \quad D(x)=\frac{2 \bar{b}}{N}\left(1-x^{2}\right)+\frac{4 a}{N} .
$$

While the drift function remains unchanged, the diffusion function, in this new setting, is N-dependent. This means that in the limit of large $N$, the fluctuations progressively attenuate, until they can become completely negligible (see paragraph 6). ${ }^{6}$

\subsection{Equilibrium Distribution}

In the following, we derive some macroscopic statistical properties of the system governed by the transitions (3) and approximated by the FPE (9) with drift and diffusion given by (10). Since $x$ is bounded between -1 and 1 , we also have to add the 'natural' boundary conditions of the model, that the probability current:

$$
j(x, t)=-2 a x \omega(x, t)-\frac{\partial}{\partial x}\left(b\left(1-x^{2}\right) \omega(x, t)\right)
$$

has to vanish at $|x|=1$. Interestingly, the parameters $a$ and $b$ do not enter directly neither into the unconditional distribution $\omega_{e}(x)$ nor into the unconditional moments, but only via their ratio $\varepsilon=a / b$.

As detailed in Appendix A.2, the following closed-form expression for $\omega_{e}(x)$ can be derived:

$$
\omega_{e}(x)=\frac{\Gamma(2 \varepsilon)}{2^{2 \varepsilon-1} \Gamma(\varepsilon)^{2}}\left(1-x^{2}\right)^{\varepsilon-1} .
$$

We recover the main findings of Kirman [1993]: $\varepsilon>1$, i.e. dominance of autonomous switches, implies uni-modality with a peak at $x=0$ and possibly small fluctuations of $\ln \left(\frac{p_{t}}{p_{f}}\right)$ around zero. Dominance of the herding component, $\varepsilon<1$, however, leads to a bimodal distribution with probability mass reaching its maximum at \pm 1 , generating phases of overvaluation and undervaluation of the price compared to its fundamental value. In the knife-edge scenario $\varepsilon=1$, the autonomous switching propensity is exactly compensated by the herding tendency, generating a uniform distribution of $x$. Note that the distribution (12) is symmetric around its mean value 0 , which reflects the underlying symmetry between the two states of the system ${ }^{7}$.

\footnotetext{
${ }^{6}$ In the limit $N \rightarrow \infty$ convergence to the equilibrium distribution, would take infinitely long time.

${ }^{7}$ Alfarano et al. [2005] derive closed-form solutions for an asymmetric herding model with different propensities for autonomous moves, $a_{1}$ and $a_{2}$, in one or the other direction. In this more general case, the resulting unconditional distribution is asymmetric, but covers eq. (12) as a special case.
} 
It is interesting to show that for $\varepsilon>>1$, the equilibrium distribution converges to a Gaussian with mean zero and variance $1 / 2 \varepsilon$. In this case, eq. (12) can be written as $^{8}$

$$
\omega_{e}(x) \rightarrow \frac{1}{\sqrt{2 \pi \sigma^{2}}} \exp \left[-\frac{1}{2}\left(\frac{x}{\sigma}\right)^{2}\right]
$$

where $\sigma^{2}=\frac{1}{2 \varepsilon}$.

If one would like to investigate the case of pure herding, $\varepsilon=0$, the boundary conditions would have to be modified in order to avoid absorbing states at $|x|=1$. Following for example Lux and Marchesi [1999], reflecting boundary conditions can be imposed so that at least $n_{0}$ agents remain always optimistic or pessimistic. Let $\phi=n_{0} / N$, the boundary conditions then require the probability current to vanish at $|x|=1-\phi$. The parameter $\phi$ can become arbitrary small for $N \rightarrow \infty$. Alfarano and Lux [2005] provide analytical results and numerical simulations of this scenario. They show that the stochastic switches between the vicinity of $x=1$ and $x=-1$ also give rise to volatility clustering, a leptokurtic distribution of returns and persistence in the level of squared and absolute returns.

\subsection{Unconditional Moments of Returns}

Using the equilibrium distribution (12) we can proceed by deriving a compact formula for the calculation of moments (cf. Appendix A.2):

$$
E\left[\left(1-x^{2}\right)^{k}\right]=2^{2 k} \frac{\Gamma(2 \varepsilon)}{\Gamma(2 \varepsilon+2 k)}\left(\frac{\Gamma(\varepsilon+k)}{\Gamma(\varepsilon)}\right)^{2} .
$$

Equipped with this result, we can invoke the Langevin equation (see paragraph 5) for the dynamics of $x$ to characterize the ensuing dynamics of relative price changes. Neglecting changes of the fundamental value, we see that eq. (7) simply leads to ${ }^{9}$ :

$$
r(t, \Delta t)=x(t+\Delta t)-x(t)=-2 a \Delta t x(t)+\sqrt{2 b \Delta t\left(1-x^{2}\right)} \eta(t+\Delta t) .
$$

${ }^{8}$ In the case $\varepsilon \gg 1$, the $x$-dependence in eq. (12) can be written as

$$
\exp \left[(\varepsilon-1) \ln \left(1-x^{2}\right)\right] \approx \exp \left[-\varepsilon x^{2}\right],
$$

where the last step follows from the approximation

$$
\ln \left(1-x^{2}\right) \approx-x^{2}, \quad \text { for } \quad|x| \ll 1 .
$$

The $\varepsilon$-dependent prefactor in eq. (12) can be simplified using Stirling's approximation for the Gamma function,

$$
\Gamma(y+1) \approx \sqrt{2 \pi y} y^{y} e^{-y}, \text { for } y \gg 1 .
$$

\footnotetext{
${ }^{9}$ Note that the arbitrary interval of time $\tau$ of eq. (7) is now replaced by the macro-time $\Delta t$, which will be defined in paragraph 5.1.
} 
Eq. (15) allows to determine the expectation of even powers of the returns, $r^{2 k}$, from those of $x^{2 k}$. Given the symmetry of the unconditional distribution, all odd moments of returns are zero. In leading order $\Delta t$ we get:

$$
E\left[r^{2 k}\right]=(2 b \Delta t)^{k} E\left[\left(1-x^{2}\right)^{k} \eta^{2 k}\right] .
$$

The second moment and kurtosis ${ }^{10} \kappa$, for example, are given by:

$$
E\left[r^{2}\right]=\frac{4 a \Delta t}{2 \varepsilon+1}, \quad \kappa=\frac{3}{\varepsilon(2 \varepsilon+3)} .
$$

Eq. (17) gives us some first insights into the potential explanatory power of this simple asset pricing model. Namely, one immediately infers that excess kurtosis is positive and vanishes only for $\varepsilon \rightarrow \infty$ so that even in the case of a uni-model distribution, the herding mechanism would lead to a leptokurtic shape of returns which is in agreement with the ubiquitous empirical deviation of relative price changes from Gaussian behavior. Since $\varepsilon \rightarrow \infty$ would imply dominance of stochastic search, due to very large $a$ compared to $b$, we observe that it is the introduction of interpersonal communication that leads to the emergence of leptokurtic returns. Leptokurtosis is, thus, a generic feature of this framework. However, it comes along with an exponential decay for large returns. Note, in fact, that eq. (16) guarantees the theoretical existence of all even moments of the return distribution; our model, therefore, does not permit a 'true' scaling for the tail of the distribution, as observed in real data.

\subsection{Autocorrelation functions}

Conditional properties of the population index $x$ and returns can be obtained in various ways. One approach could be based on the general solution to the Fokker-Planck equation, which can be derived as a series expansion of Jacobi polynomials (see Alfarano [2005]). We can, then, provide a complete characterization of conditional moments for any transformation of returns. Alternatively, correlation functions could be determined recursively using the Langevin approximation to the Fokker-Planck equation. Although this approach has severe limitations in dealing with non-linear transformations of returns, it can be employed to compute the autocorrelation for raw and squared returns.

To this end, we consider the discrete time steps $t=n \Delta t$ and define the recursion:

$$
F_{n}=E\left[x_{t} \cdot x_{0}\right]
$$

\footnotetext{
${ }^{10}$ We define the kurtosis as

$$
\kappa=\frac{E\left[r^{4}\right]}{E\left[r^{2}\right]^{2}}-3
$$
}


for which the Langevin equation provides us with the difference equation:

$$
F_{n}=(1-2 a \Delta t) F_{n-1} .
$$

Backward iteration leads to:

$$
F_{n}=F_{0}(1-2 a \Delta t)^{n}
$$

with $F_{0}=E\left[x_{0}^{2}\right]$, which can be used to determine the auto-correlation $C_{x}(t)$ of $x$ :

$$
C_{x}(t)=(1-2 a \Delta t)^{\frac{t}{\Delta t}} .
$$

In the limit $\Delta t \rightarrow 0$, eq. $(18)$ becomes $C_{x}(t)=\exp (-2 a t)$. This result is not surprising given the linear drift function of the F-P equation (see Gardiner [2003], Van Kampen [1992]).

The correlation of $r_{t}$ can similarly be expressed in terms of $F_{n}$ :

$$
E\left[r_{t} \cdot r_{0}\right]=E\left[\left(x_{t+\Delta t}-x_{t}\right) \cdot\left(x_{\Delta t}-x_{0}\right)\right]=2 F_{n}-F_{n-1}-F_{n+1},
$$

which leads to the expression (see Appendix A.3):

$$
C_{r}(t)=-a \Delta t(1-2 a \Delta t)^{\frac{t}{\Delta t}-1} \approx-a \Delta t \exp (-2 a t) .
$$

In principle, the small negative correlation is at odds with the informational efficiency and martingale nature of financial prices. However, the small mean-reverting tendency of the population index and returns can easily be blurred by the noise level generated by the finiteness of the data sample and would not easily be detected if $a$ were not too large (typical values are of the order of magnitude of $a \approx 10^{-2}$ ).

One can proceed similarly in deriving the auto-correlation functions of higher moments. In order to compute $C_{r^{2}}(t)$ one starts with the relation ${ }^{11}$ :

$$
E\left[r_{t}^{2} \cdot r_{0}^{2}\right]=4 b^{2} \Delta t^{2}\left(1-E\left[x_{t}^{2}\right]-E\left[x_{t}^{2} \eta_{0}^{2}\right]+E\left[x_{t}^{2} x_{0}^{2} \eta_{0}^{2}\right]\right)
$$

After some efforts, we finally arrive at:

$$
C_{r^{2}}(t)=\frac{1}{4 \varepsilon^{2}+6 \varepsilon+3} \exp (-2 b t(2 \varepsilon+1)) .
$$

Obviously, $C_{r^{2}}(t)>0$ for all $t>0$ so that we have shown that volatility persistence, as the second universal time series property of returns, is also a natural outcome of this model.

\footnotetext{
${ }^{11}$ Additional details are to be found in Appendix A.3.
} 


\subsection{Mean First Passage Time}

It is interesting to complement the analysis of conditional properties by closed-form solutions of so-called mean first passage times (MFPT), defined as the time the system takes on average to switch from one state to another. Of major interest for the calculation of MFPTs are the modes and anti-modes $x=0,-1$ and 1 . As shown in Appendix A.4, in the case $0<\varepsilon<1$ the MFTP between the two modes $x=1$ and $x=-1$ is given by:

$$
T(-1 \rightarrow 1)=T(1 \rightarrow-1)=\frac{1}{b} \frac{\pi}{1-2 \varepsilon} \frac{\cos (\pi \varepsilon)}{\sin (\pi \varepsilon)} .
$$

As can be seen, $T(\cdot)$ diverges for $\varepsilon \rightarrow 0$, which corresponds to the emergence of two absorbing states at $|x|=1$ in the case of a vanishing autonomous switching probability. The lock-in at one of the extremal modes from which the dynamics would not return, since $a=0$ and $\varepsilon=0$, is mirrored in a divergence to infinity of its MFPT. For $\varepsilon \rightarrow 1$, divergence is obtained because for the uni-model case established for $\varepsilon>1, \pm 1$ are reached with a vanishing probability. Interestingly and maybe running against the first intuition, the MFPT is not monotonic in $\varepsilon$, but has a minimum at $\varepsilon=0.5$, i.e. $a=0.5 b$. At $\varepsilon=0.5$, an increase of either the autonomous component or the herding propensity would lead to an increase of $T(\cdot)$.

Remark. If we move to the extensive formulation (1), all the previous equations still hold, but would contain a dependency on the number of agents via the relation $b=\frac{\bar{b}}{N}$. Moreover, the parameter $\varepsilon$ has to be replaced by the following $\mathrm{N}$-dependent parameter:

$$
\varepsilon_{N}=N \frac{a}{\bar{b}}
$$

Obviously, volatility dependence gets lost with an increasing population size, as it is apparent from eq. (21). The vanishing heteroscedasticity is reflected in a convergence to zero of the autocorrelation of squared returns. The loss of volatility dependence corresponds to the approach of the unconditional distribution towards the Gaussian, as illustrated in Figure 2. The stylized facts, namely leptokurtic distribution of return and volatility clustering measured as the non-vanishing correlation in the squared returns, do not survive when we increase the number of agents in the market. This means that the herding mechanism depending on the fraction of agents in the other group, at least in the present setting, might not be a suitable mechanism to explain the stylized facts as emerging properties of interactions among the traders. On the contrary, the model with dependence on the overall group occupation numbers, as formalized in eq. (3), does not exhibit any relevant influence of the system size, and, therefore, gives identical results for markets with any number of participants. 


\section{Different Avenues for Simulating the Herding Model}

\subsection{Occupation number approach}

Following multi-particle simulations in statistical physics, different avenues exist for simulating the above model. The first, obvious choice would be a true microscopic simulation keeping track of the state of each individual agent and determining its switches over time by random number draws. Of course, the continuous-time framework would have to be simulated in discretised form. As an obvious restriction, these simulations would have to observe the condition that the sum of all transition probabilities has to remain smaller than unity, i.e. $\sum \pi\left(n \rightarrow n^{\prime}\right) \Delta t_{0} \leq 1$ which translates into an upper bound for the usable micro time steps $\Delta t_{0}$ depending on the size of the population:

$$
\triangle t_{0} \leq \frac{2}{N(2 a+b N)},
$$

since the herding component in both transition rates assumes its maximum at $n=\frac{N}{2}$. The maximum admissible time increment obviously decreases hyperbolically with the population size, $\Delta t_{0} \propto N^{-2}$. Microscopic simulations, therefore, become increasingly more time consuming with larger population size.

As an alternative, we could resort to simulating the aggregated outcome of the stochastic dynamics in terms of the population configuration which is summarized by the variable $n$, without taking into account the information on the history of every single individual. Rewriting (3) in terms of this state variable, one can see that transitions between states occur with probabilities:

$$
\rho\left(n^{\prime}, t+\Delta t_{0} \mid n, t\right)=\left\{\begin{array}{cc}
2 \frac{(N-n)(a+b n)}{N(2 a+b N)} & \text { if } n^{\prime}=n+1 \\
2 \frac{n(a+b(N-n))}{N(2 a+b N)} & \text { if } n^{\prime}=n-1 \\
b \frac{N^{2}-4 n(N-n)}{N(2 a+b N)} & \text { if } n^{\prime}=n \\
0 & \text { otherwise }
\end{array}\right.
$$

In $(23), \Delta t_{0}$ had been chosen such that it allows the highest "efficiency" of the macroscopic simulations, i.e. such that it minimizes the probability to observe no change in $n$. This is equivalent to using (22) as an equality. Of course, only the smallest possible change in $n$ can be observed during the micro-step $\Delta t_{0}$ of the simulation. Similarly as in Kirman and Teyssière [2002], we may, therefore, introduce a distinction between micro time steps $\Delta t_{0}$ and macro time increments $\Delta t$ in which many changes of $n$ may be observed. The difference is, however, that we also allow the price process to operate along the micro time scale and that together with the change of the population configuration we also aggregate over many small price changes at the macro time scale $\Delta t$. To illustrate the dynamics and to provide a justification of a "useful" macro time scale, consider the following scenario: for $n=0$, 
the system can evolve like follows: it may remain unchanged with probability $\frac{N}{N+2 \varepsilon}$, or it may change by one unit with the small probability $\frac{2 \varepsilon}{N+2 \varepsilon}, \varepsilon=\frac{a}{b}$. Therefore, the average number of iterations of (23) needed to observe a move of one agent is approximately equal to $N$. To observe larger increments of $n$ (as changes by one unit are negligible, in particular for the case of large $N$ ), we need a multiple of $N$ to define the number of micro time steps that make up one macro interval. From eq. (22), a sensible choice appears to be:

$$
\Delta t=\frac{b}{2} N^{2} \Delta t_{0}
$$

In the simulations, one then iterates the process $\frac{b}{2} N^{2}$ times until one stores the current value of $n$ as one realization at the macroscopic time scale $\Delta t$. The most interesting aspect of this approach is that it guarantees invariance of the dynamics of the macroscopic variable $n$ with respect to the number of agents due to the flexibility of the chosen macro time scale. This does not come as a surprise since we have already noticed that the drift and diffusion functions in the FPE (9) are independent of $N$. Figure 1 provides an illustration of this feature in which we indeed observe no qualitative difference in the behavior of time series for different sizes of the population.

All in all, in order to have a meaningful micro-simulation algorithm for the simulation of the Markov chain governed by eq. (3) in the case of various population sizes, we have to introduce two different time scales. Note that the scaling of the macro-time with the number of individuals is non-linear. Again the non-linear dependence on $N$ of the macro time-scaling reflects the non-extensivity of the transition probabilities (3).

\subsection{Concentration approach}

A very different behavior is observed when the system is described by extensive transition rates (1) in the limit $N \rightarrow \infty$. Let us start with the reference parameters $\frac{a_{0}}{N_{0}}$ (we assume that $a_{0}=0.5$ ) for the autonomous switching term, and $\bar{b}=1$ for the herding parameter; $N_{0}$ is the starting population size. A glance at the diffusion function in eq. (10) shows that the effective herding parameter (the constant in front of the quadratic term) is $\frac{\bar{b}}{N}$, for a market populated by $N$ traders. The ratio between the autonomous term and the herding parameter is, then, defined by:

$$
\varepsilon_{N}=\frac{N}{N_{0}} \frac{a_{0}}{\bar{b}}=\frac{N}{2 N_{0}} .
$$

When the number of agents trading in the market $N$ coincides with the reference size $N_{0}$, the market falls into the regime dominated by the herd behavior, with a bimodal probability density function for $x$. If one now increases the number of 

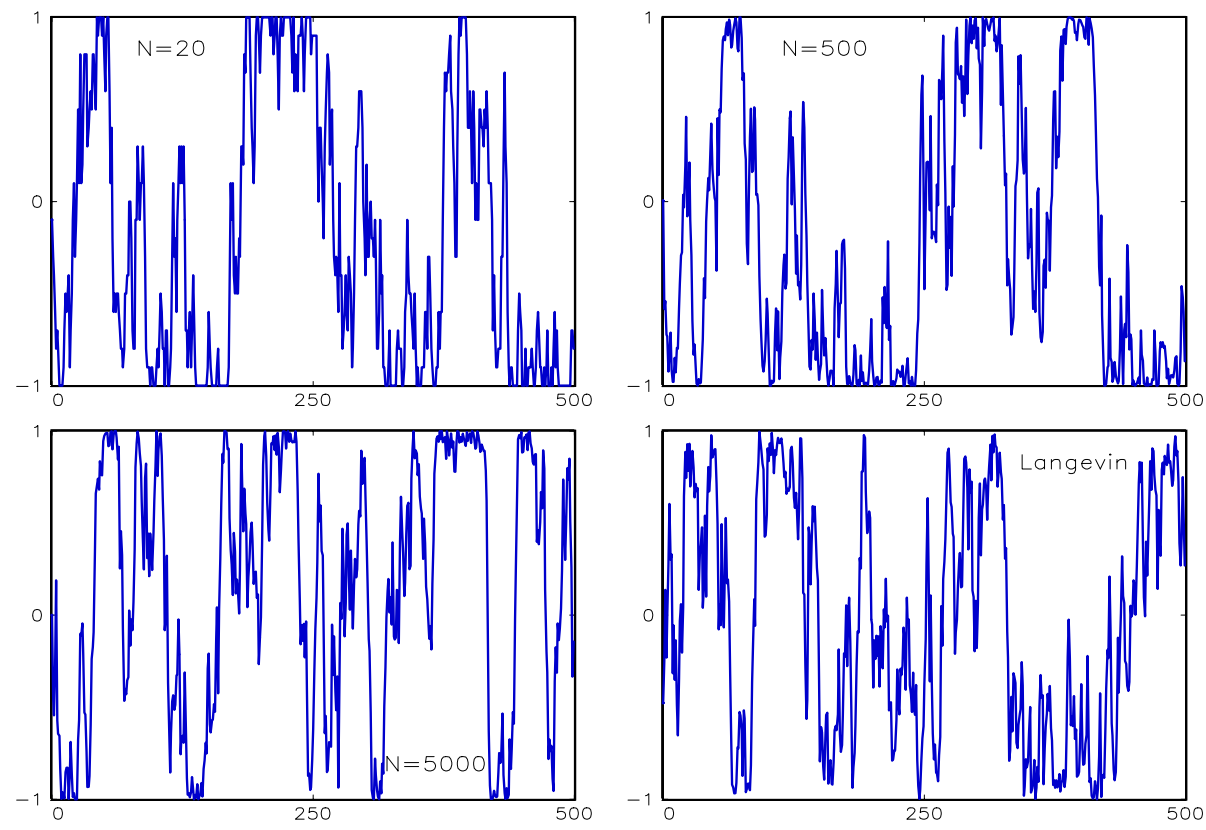

Figure 1: The four panels show snapshots of the dynamics of the population index for increasing values of the number of agents $N$. The right bottom panel shows a trajectory computed via the Langevin approach given by eq. (29). The other three panels show different examples of the simulation of the model with transition rates according to eq. (3), as detailed in section 5.1.

agents over the starting level $N_{0}$ and, at the same time, leaves unchanged the other parameters, the equilibrium distribution will undergo a transition from a bimodal shape to a narrowly peaked distribution around the average value $E[x]=0$. The linear dependence of $\varepsilon_{N}$ on the number of agents $N$, cf. eq. (25), brings about a convergence of the equilibrium distribution $\omega_{e}(x)$ to a Gaussian, as analytically derived in eq. (13) and illustrated in Figure 2. The transition from a bimodal to a unimodal distribution implies the existence of a critical number of agents $N_{C}$, which we might conveniently define by the relation ${ }^{12}$ :

$$
\varepsilon_{N_{C}}=\frac{N_{C}}{N_{0}} \frac{a_{0}}{\bar{b}}=1 \quad, \quad N_{C}=N_{0} \frac{\bar{b}}{a_{0}} .
$$

\footnotetext{
${ }^{12}$ Note that in the case $\varepsilon_{N}=1$, the equilibrium distribution is uniform.
} 
The critical size of the market, $N_{C}$, might be used to distinguish between two regimes. An interacting-agent regime, where the random meetings among agents have a relevant impact on the opinion index dynamics, with a resulting bimodal distribution, and an individualistic regime, where the market behaves like a collection of independent agents and the interactive component has a negligible influence. The resulting Gaussian probability distribution for the case $N \gg N_{C}$ is, in fact, the asymptotic distribution for a collection of independent agents. In the case of extensive transition rates and a large number of components the system can be described by a deterministic equation for the mean and a 'small' superimposed noise perturbation (see Gardiner [2003] and, in particular, Aoki [1996, 2002] for several applications of this formalism to economics). It is well known that the following equation holds
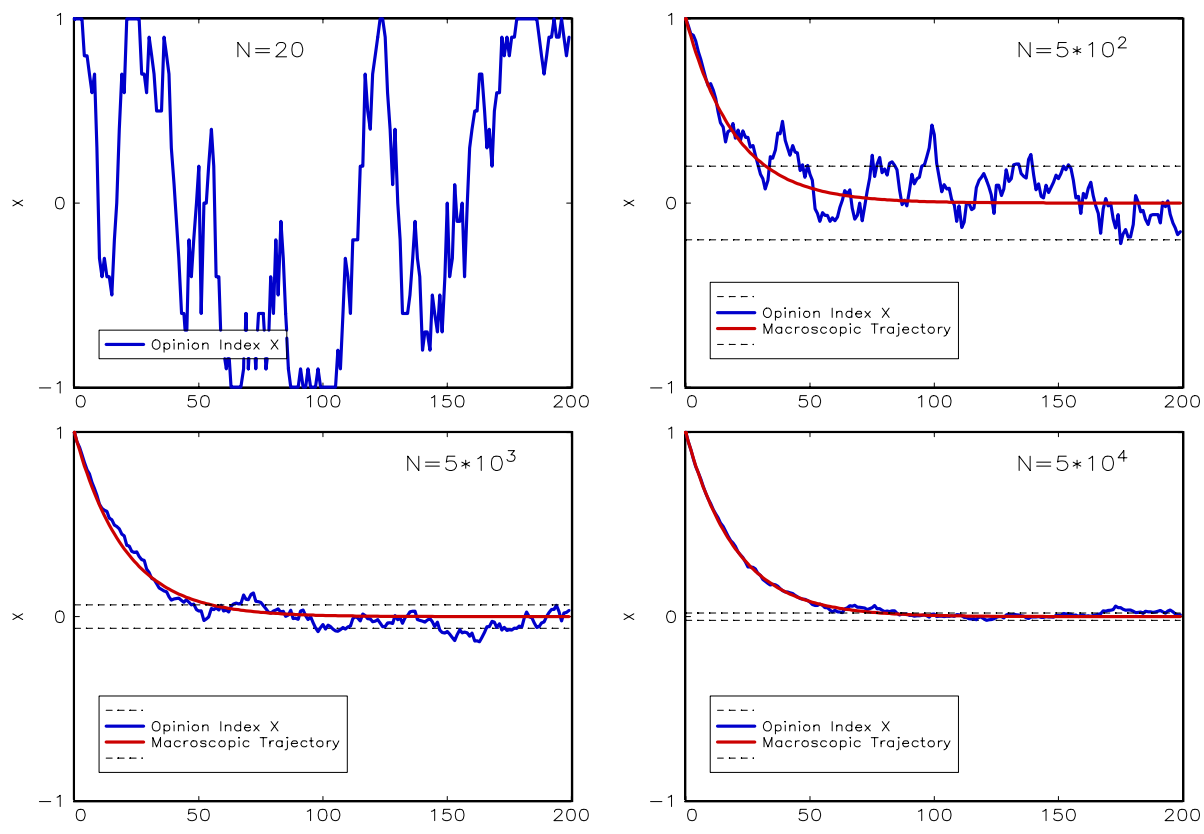

Figure 2: The four panels show the emergence of the macroscopic skeleton of the system when governed by the extensive transition probabilities (1), for an increasing number of agents. Note the clear exponential decay of the trajectory of $x$ for large population size, in accordance with the deterministic eq. (28). The dashed lines represent the standard deviation of the equilibrium distribution of the variable $x$ (see appendix A.5). The underlying parameters are $N_{0}=20, \bar{b}=1$ and $a_{0}=0.5 / N_{0}$. 
for the mean value $\bar{x}(t)$ of a stochastic process with linear drift function:

$$
\frac{d}{d t} \bar{x}(t)=A(\bar{x}(t))
$$

where $A(\cdot)$ is given by eq. (11) in our case. Equation (27) simply states that the mean of $\omega(x, t)$ follows a differential equation of first order, whose unique and fully deterministic solution is given by:

$$
\bar{x}(t)=\bar{x}_{0} \exp (-2 \varepsilon b t),
$$

where $\bar{x}_{0}$ is the mean value of the starting distribution $\omega_{0}(x, 0)$. As a result, $\bar{x}$ converges to its unconditional value $E[x]=0$ with an exponential rate. If we take into account the example of Figure 1, the macroscopic equation predicts no evolution for $\bar{x}$, since the starting value $\bar{x}_{0}$ coincides with the unconditional expected value. On the contrary, we observe a non trivial dynamics of opinion index $x$ for every size of the agents population, which gives rise to the interesting statistical properties of our artificial market. The contrast between the macroscopic approximation of eq. (27) and the more complete description in terms of the FPE is striking. The endogenous fluctuations of the system might be neglected at a cost of a meaningless description of its time-evolution. On the contrary, the approach to the equilibrium distribution for the model governed by intensities rather than occupation number is well described by eq. (28), as illustrated in Figure 2. In the case of extensive transition probabilities and a large number of agents, the aggregate behavior of the system can be described by a deterministic equation for the mean and vanishing fluctuations around $\bar{x}(t)$ for $N \rightarrow \infty$.

\subsection{Langevin approach}

Keeping with the non-extensive formulation of eq. (3), one might, as a third alternative, simulate the model using a Langevin equation providing a Gaussian approximation to the stochastic dynamics over $\Delta t / \Delta t_{0}$ micro time steps per time unit $\Delta t$ using drift and diffusion terms derived below (cf. eq 10):

$$
x(t+\Delta t)=(1-2 a \Delta t) x(t)+\sqrt{2 b \Delta t\left(1-x^{2}\right)} \eta(t) .
$$

In eq. (29), $\eta(t)$ is an iid noise variable following a Normal distribution with mean zero. In Appendix A.6, we show how (29) can be derived from the Fokker-Planck equation if $\Delta t$ is small enough to justify negligence of terms of order $(a \Delta t)^{2}$ and $(b \Delta t)^{2}$. Although for small step sizes $\Delta t$, the Langevin equation indeed provides a close approximation to the underlying agent-based model, it has the drawback that it might violate the built-in boundaries $x \in[-1,1]$ of the population dynamics. To keep $x$ within meaningful bounds in simulations of (29), reflecting boundaries at $x= \pm 1$ need, therefore, to be imposed by hand. Despite these difficulties, (29) has 
the important advantage that it not only facilitates numerical simulations, but also enables us to compute conditional and unconditional moments of the price process. In Figure 1 we can observe the qualitatively good agreement between the microsimulation based on eq. (23) and the Langevin approach.

\section{Local vs non-local interactions}

Extensive transition rates describe systems whose evolution is driven by 'local' mechanisms. In order to justify this assertion, namely the relation between the extensive transition probabilities with the local character of the interactions, we will provide some qualitative arguments. Let us start considering a system composed of a large number of independent components. To describe the asymptotic behavior of such a system, we may advocate the CLT, expecting Gaussian fluctuations around the mean of some aggregate quantities. The ratio of the amplitude of the fluctuations relative to their mean, then, will converge to zero. More precisely, we expect a dependence of the type $O\left(\frac{1}{\sqrt{N}}\right)$. The convergence to a well-defined mean with vanishing fluctuations is the basic justification for a deterministic approach for the description of models composed by independent 'particles' in terms of macroscopic observables.

This scenario can be generalized to include some sort of interactions among the constituents, which typically generate correlations among the different particles. If the correlations are 'weak' enough, we can still apply the CLT and arrive at the Gaussian regime for the fluctuations like for independent particles, with a well defined mean for aggregate quantities, which can still be described by deterministic laws. Here the term 'weak' stands for a limited number of constituents involved in the interaction mechanism. On the contrary, the mechanism has a 'non-local' or, in extreme cases like our simple herding model, global character if the range of the correlations involves a macroscopic fraction of elements. The long-range correlations prevent the use of the CLT and, therefore, we cannot expect the asymptotic Gaussian regime for the fluctuations. Spatial or temporal correlations in the level of fluctuations might be observed for any system size. Extensive transition rates guarantee convergence to the Gaussian regime, while non-extensive systems might or might not exhibit convergence to the Gaussian regime. Finally, we should stress here that the classification between the local or non-local character of the interactions is based on the interpretation of the different asymptotic behavior under aggregation in the limit of a large number of constituents, which can be inferred from the FPE.

The somewhat counterintuitive result here is that, on one hand, pair-wise meetings and interactions in the line with the original philosophy of random meetings 
in space -eq. (3)- lead to a global character of the system with the dynamics preserving the interesting features independent on the system size. Dependence of the transitions on overall fractions of groups within the population, on the other hand, lead to a more 'local' character of the dynamics and loose all interesting features with an increasing number of agents, ultimately converging to a Gaussian regime. The solution of this puzzle is that in the non-extensive formalization the number of neighbors per constituent increases with an increasing population. It means that every agent is coupled with the same fraction of other agents for all system size, which guarantees that the interesting properties of the fluctuations are independent on $N$. Hence, the intensity of the interpersonal coupling is preserved via eq. (3) for varying numbers of agents $N$. In the extensive framework of eq. (1), in contrast, the herding component looses its importance vis-à-vis the autonomous switches when increasing the number of agents, because the coupling intensity effectively decreases with system size.

\section{Conclusion}

The aim of the present paper was to contribute to a better understanding of the statistical properties of agent-based models of financial markets. To this end, we attempted a thorough analysis of different types of herding models in the tradition of Kirman's seminal ant model. Our novel insights are the following:

(i) We were able to derive closed-form solutions for various moments as well as the autocorrelations of raw and squared returns. Our results demonstrate that both leptokurtosis of returns and temporal dependence of volatility are generic features of this model and are intimately linked to the herding component. This underscores the importance of boundedly rational behavior as potential explanation of the stylized facts. Admittedly, our model did not produce power laws (hyperbolic decay) of the cumulative distribution nor of the autocorrelation function. However, in a companion paper, (Alfarano and Lux [2005]), we also show that pseudo-empirical analysis of time series for such a model could easily produce 'spurious' or 'apparent' power laws, which is a typical outcome within a preasymptotic regime with a length of the time series below some threshold value.

(ii) We have also investigated the dependency of the 'interesting' dynamics and stylized facts on system size, revisiting earlier findings of trivial large $N$ limit in similar models. As it turns out, trivial or non-trivial behavior of large markets depend on the exact formalization of transition rates. Ironically, a literal interpretation of

pair-wise interactions leads to some sort of 'global' coupling of agents, which preserves all interesting features of the model for any system size. On the contrary, a more conventional formalization of the transitions rates in terms of concentration 
leads to a convergence to uninteresting (and unrealistic) Gaussian dynamics in the limit of large markets.

\section{References}

S. Alfarano. An Agent-based Stochastic Volatility Model. PhD thesis, Department of Economics, University of Kiel, 2005. In preparation.

S. Alfarano and T. Lux. A noise trader model as a generator of apparent financial power laws and long memory. Macroeconomics Dynamics, 2005. in press.

S. Alfarano, T. Lux, and F. Wagner. Estimation of agent-based models: the case of an asymmetric herding model. Computational Economics, 26:19-49, 2005.

M. Aoki. New Approaches to Macroeconomic Modeling: Evolutionary Stochastic Dynamics, Multiple Equilibria, and Externalities as Field Effects. University Press, Cambridge, 1996.

M. Aoki. Modeling Aggregate Behavior and Fluctuations in Economics. University Press, Cambridge, 2002.

J. Arifovic. The behaviour of the exchange rate in the genetic algorithm and experimental economies. Journal of Political Economy, 104:510-541, 1996.

W. B. Arthur, J. H. Holland, B. LeBaron, R. Palmer, and P. Tayler. Asset pricing under endogenous expectations in an artificial stock market. Economic Notes, 26: 297-330, 1997.

E. Barucci. Financial Markets Theory Equilibrium, Efficiency and Information. Springer, 2003.

A. Beja and M. B. Goldman. On the dynamic behavior of prices in disequilibrium. Journal of Finance, 35:235-248, 1980.

D. Challet and M. Marsili. From minority game to the real markets. Physical Review E, 68:168-176, 2004.

S. H. Chen and C. H. Yeh. On the emergent properties of artificial stock markets: The Efficient Market Hypothesis and the Rational Expectations Hypothesis. Journal of Economic Behavior and Organization, 49:217-239, 2002.

R. H. Day and W. Huang. Bulls, bears, and market sheep. Journal of Economic Behavior and Organization, 14:299-329, 1990.

P. DeGrauwe, Dewachter H, and M. J. Embrechts. Exchange Rate Theory: Chaotic Models of Foreign Exchange Market. Blackwell, Oxford, 1993. 
E. Egenter, T. Lux, and D. Stauffer. Finite-size effects in Monte Carlo simulations of two stock market models. Physica A, 268:250-256, 1999.

H. Föllmer, U. Horst, and A. Kirman. Equilibria in financial markets with heterogeneous agents: A probabilistic perspective. Journal of Mathematical Economics, 41:123-155, 2005.

C. W. Gardiner. Handbook of Stochastic Methods for Physics, Chemistry and the Natural Sciences. Springer, 2003. Third edition.

E. Grannen and G. Swindle. Contrarians and volatility clustering. Complex Systems, 8:75-89, 1994.

U. Horst. Financial price fluctuations in a stock market model with many interacting gents. Economic Theory, 25(4):917-932, 2004.

A. Kirman. Epidemics of opinion and speculative bubbles in financial markets. In M. P. Taylor, editor, Money and Financial Markets, pages 354-368. Blackwell, Cambridge, 1991.

A. Kirman. Ants, rationality, and recruitment. Quarterly Journal of Economics, 108:137-156, 1993.

A. Kirman and G. Teyssière. Microeconomic models for long memory in the volatility of financial time series. Studies in Nonlinear Dynamics 83 Econometrics, 5:137$156,2002$.

B. LeBaron, W. B. Arthur, and R. Palmer. The time series properties of an artificial stock market. Journal of Economic Dynamics and Control, 23:1487-1516, 1999.

M. Levy, H. Levy, and S. Solomon. A microscopic model of the stock market: Cycles, booms, and crashes. Economics Letters, 45:103-111, 1994.

T. Lux. Herd behaviour, bubbles and crashes. Economic Journal, 105:881-896, 1995.

T. Lux. Time variation of second moments from a noise trader/infection model. Journal of Economic Dynamics and Control, 22:1-38, 1997.

T. Lux. The socio-economic dynamics of speculative markets: Interacting agents, chaos, and the fat tails of return distributions. Journal of Economic Behavior and Organization, 33:143-165, 1998.

T. Lux and M. Marchesi. Scaling and criticality in a stochastic multi-agent model of a financial market. Nature, 397:498-500, 1999. 
T. Lux and M. Marchesi. Volatility clustering in financial markets: A microsimulation of interacting agents. International Journal of Theoretical and Applied Finance, 3:67-702, 2000.

T. Lux and S. Schornstein. Genetic learning as an explanation of stylized facts of foreign exchange markets. Journal of Mathematical Economics, 41:169-196, 2005.

M. O’Hara. Market Microstructure Theory. Blackwell, Cambridge, 1995.

J. B. Ramsey. On the existence of macro variables and of macro relationships. Journal of Economic Behavior and Organisation, 30:275-299, 1996.

N. G. Van Kampen. Stochastic processes in Physics and Chemistry. North Holland, Amsterdam, 1992. Revised edition.

\section{A Appendix}

\section{A.1 Derivation of the Fokker-Planck Equation}

In this appendix, we derive the Fokker-Plank equation (9) as a second-order Taylor approximation to the continuum of our population dynamics governed by eq. (3). In order to do so, we introduce the intensive variable

$$
x=2 n / N-1,
$$

that, if the total number of agents $N$ is large enough, can be treated as a continuous quantity. The relation between the transition rates (3), expressed in terms of the variables $n$ or $x$, is given by the following formula:

$$
\pi_{n}^{ \pm}=\frac{N^{2}}{4} \pi_{x}^{ \pm}
$$

Note that the probabilities are invariant under the transformation (A1), therefore $\bar{\omega}_{n}=\bar{\omega}_{x}$. The transition rates $(3)$ are now functions ${ }^{13}$ of $x$ :

$$
\begin{aligned}
& \pi_{x}^{+}=(1-x)(2 a / N+b(1+x)), \\
& \pi_{x}^{-}=(1+x)(2 a / N+b(1-x)) .
\end{aligned}
$$

Let us introduce the "step" operators $\mathbf{E}$ and $\mathbf{E}^{-1}$, using the notation of Van Kampen [1992]. Their effects on an arbitrary function $f(n)$ are respectively to decrease or increase its integer argument $n$ by one unit. Formally:

$$
\mathbf{E}[f(n)]=f(n+1) \quad, \quad \mathbf{E}^{-1}[f(n)]=f(n-1) .
$$

\footnotetext{
${ }^{13}$ The new transitions have to be read as $\pi_{x}^{ \pm}=\pi(x \rightarrow x \pm 2 / N)$.
} 
With the aid of the operators $\mathbf{E}[\cdot]$ and $\mathbf{E}^{-1}[\cdot]$, the Master equation (8) for the onestep process can be compactly rewritten in terms of the variable $x$ :

$$
\frac{\partial}{\partial t} \bar{\omega}_{x}=(\mathbf{E}-1)\left[\pi_{x}^{-} \frac{N^{2}}{4} \bar{\omega}_{x}\right]+\left(\mathbf{E}^{-1}-1\right)\left[\pi_{x}^{+} \frac{N^{2}}{4} \bar{\omega}_{x}\right],
$$

where we skip the obvious time dependence of $\bar{\omega}_{x}$. Considering the continuous limit for $x$, the probability $\bar{\omega}_{x}$ has to be 'converted' into a probability density:

$$
\bar{\omega}_{n}=\bar{\omega}_{x} \equiv \omega_{x} \frac{N}{2},
$$

where $\omega_{x}$ is a continuous function of $x$. The probability density might be alternatively defined by the limit:

$$
\omega_{x}=\lim _{N \rightarrow \infty} \frac{\bar{\omega}_{x}}{\Delta x}=\lim _{N \rightarrow \infty} \frac{N}{2} \bar{\omega}_{x} .
$$

Eq. (A2) can be rewritten as:

$$
\frac{\partial}{\partial t} \omega_{x}=\frac{N^{2}}{4}(\mathbf{E}-1)\left[\pi_{x}^{-} \omega_{x}\right]+\frac{N^{2}}{4}\left(\mathbf{E}^{-1}-1\right)\left[\pi_{x}^{+} \omega_{x}\right] .
$$

Since in the limiting case, the step operator acts on continuous functions, we can perform the Taylor expansion:

$$
\mathbf{E}[f(x)]=f(x+\Delta x)=f(x)+\Delta x \frac{d}{d x} f(x)+\frac{1}{2} \Delta x^{2} \frac{d^{2}}{d x^{2}} f(x)+o\left(\Delta x^{2}\right),
$$

where $\Delta x=2 / N$. The step operator can be approximated by the following expansion:

$$
\mathbf{E}=1+\Delta x \frac{\partial}{\partial x}+\frac{1}{2} \Delta x^{2} \frac{\partial^{2}}{\partial x^{2}}+o\left(\Delta x^{2}\right) .
$$

Note that the derivatives in eq. (A3) are replaced by the partial derivatives in eq. (A4), since the function $f$, which the operator is applied to, might have more than one variable. The expansion for the operator $\mathbf{E}^{-1}$ is simply obtained from the previous formula replacing $\Delta x$ with $-\Delta x$. Using the expansion (A4) for $\mathbf{E}$ and $\mathbf{E}^{-1}$ up to second order ${ }^{14}$, we end up with:

$$
\frac{\partial}{\partial t} \omega_{x}=\frac{N^{2}}{4}\left\{-\Delta x \frac{\partial}{\partial x}\left[\left(\pi_{x}^{+}-\pi_{x}^{-}\right) \omega_{x}\right]+\frac{1}{2} \Delta x^{2} \frac{\partial^{2}}{\partial x^{2}}\left[\left(\pi_{x}^{+}+\pi_{x}^{-}\right) \omega_{x}\right]\right\} .
$$

The $N^{2} / 4$ factor in front of the equation disappears, and we arrive finally at the Fokker-Planck equation:

$$
\frac{\partial}{\partial t} \omega_{x}=-\frac{\partial}{\partial x} A(x) \omega_{x}+\frac{1}{2} \frac{\partial^{2}}{\partial x^{2}} D(x) \omega_{x}
$$

\footnotetext{
${ }^{14}$ Higher terms would be $o\left(1 / N^{2}\right)$, and are, therefore, negligible. The reason to include the second order will be clarified later.
} 
where the drift and diffusion functions are respectively given by:

$$
A(x)=\frac{N}{2}\left(\pi_{x}^{+}-\pi_{x}^{-}\right)=-2 a x,
$$

and

$$
D(x)=\pi_{x}^{+}+\pi_{x}^{-}=2 b\left(1-x^{2}\right)+\frac{4 a}{N} .
$$

For finite $b$ the term proportional to $a / N$ can be neglected.

It should be emphasized that the Fokker-Planck equation is an approximation to the continuum of our intrinsically discrete stochastic process governed by the transition probabilities (3), up to terms proportional to $1 / N^{2}$. This equation, therefore, provides a valid approximation only if $\Delta x \ll|1 \pm x|$, i.e. not too close to the boundaries, where the discreteness of the variable $x$ cannot be neglected any longer and the term $4 a / N$ should be taken into account. More precisely, the granularity term can be neglected if $2 b\left(1-x^{2}\right) \gg(4 a / N)$, which is equivalent to the constraint $\frac{\varepsilon}{2 N}-1 \ll x \ll 1-\frac{\varepsilon}{2 N}$ However, given the dependence of $\Delta x$ on $N$, this 'critical' region might be set to any arbitrary small interval by simply increasing the total number of agents $N$.

\section{A.2 Unconditional distribution of $x$ and returns}

The following textbook formula allows to compute the equilibrium distribution $\omega_{e}(x)$, given in eq. (12) in the main text, if we know the diffusion and drift functions:

$$
\omega_{e}(x)=\frac{K}{D(x)} \exp \left(\int^{x} \frac{2 A(y)}{D(y)} d y\right)
$$

(see for instance Van Kampen [1992]). The first step consists in solving the following integral:

$$
\int \frac{2 A(x)}{D(x)} d x=-\frac{2 a}{b} \int \frac{x}{1-x^{2}} d x
$$

where we take the drift and diffusion functions from eqs. (A6) and (A7), neglecting the last term in $D(x)$. With the aid of $\varepsilon=\frac{a}{b}$ and a little effort, the solution is:

$$
\int \frac{2 A(x)}{D(x)} d x=\varepsilon \ln \left(1-x^{2}\right),
$$

that plugged into (A8), gives the equilibrium distribution $\omega_{e}(x)$ :

$$
\omega_{e}(x)=K\left(1-x^{2}\right)^{\varepsilon-1} .
$$

The constant $K$ is computed via the normalization condition:

$$
K \int_{-1}^{+1} \omega_{e}(x) d x=1 .
$$


Recalling the definition of the Beta function,

$$
B\left(\varepsilon_{1}, \varepsilon_{2}\right):=\int_{0}^{1} z^{\varepsilon_{1}-1}(1-z)^{\varepsilon_{2}-1} d z=\frac{\Gamma\left(\varepsilon_{1}\right) \Gamma\left(\varepsilon_{2}\right)}{\Gamma\left(\varepsilon_{1}+\varepsilon_{2}\right)},
$$

we obtain for symmetric coefficients $\varepsilon_{1}=\varepsilon_{2}$ and with the substitution $z=\frac{1}{2}(1+x)$ :

$$
\int_{-1}^{+1}\left(1-x^{2}\right)^{\varepsilon-1} d x=2^{2 \varepsilon-1} \frac{\Gamma(\varepsilon)^{2}}{\Gamma(2 \varepsilon)} .
$$

Solving the integral (A10), we arrive at $K=\frac{\Gamma(2 \varepsilon)}{2^{2 \varepsilon-1} \Gamma(\varepsilon)^{2}}$.

Using eq. (A9) we can derive a compact formula for the calculation of moments:

$$
E\left[\left(1-x^{2}\right)^{k}\right]=\frac{\Gamma(2 \varepsilon)}{2^{2 \varepsilon-1} \Gamma(\varepsilon)^{2}} \int_{-1}^{1}\left(1-x^{2}\right)^{\varepsilon-1}\left(1-x^{2}\right)^{k} d x .
$$

Via the definition of the Beta function, eq. (A11) becomes:

$$
E\left[\left(1-x^{2}\right)^{k}\right]=2^{2 k} \frac{\Gamma(2 \varepsilon)}{\Gamma(2 \varepsilon+2 k)}\left[\frac{\Gamma(\varepsilon+k)}{\Gamma(\varepsilon)}\right]^{2}
$$

which is used in the main text for computing the unconditional moments of returns.

\section{A.3 Autocorrelation functions}

Closed-form approximate solutions for the auto-covariances of transformations of the variable $x$ may be obtained via different approaches.

Starting from the Fokker-Plank equation (A5), we can solve for the conditional probability $\omega\left(x, t \mid x_{0}, 0\right)$, given the initial distribution $\omega_{0}\left(x_{0}\right)$. It turns out that $\omega\left(x, t \mid x_{0}, 0\right)$ can be expanded in Jacobi polynomials, which allows to compute the autocorrelation for any transformation $f(x)$ of the variable $x$ in series of these polynomials (see Alfarano [2005]).

Alternatively, correlation functions can be determined recursively using the Langevin approximation of the Fokker-Planck equation. We proceed here using this second approach. For discrete time steps $t=n \Delta t$, we consider first

$$
F_{n}=E\left[x_{t} \cdot x_{0}\right], \quad F_{0}=E\left[x_{t}^{2}\right] .
$$

From the Langevin equation (29), we obtain a recursion for $F_{n}$ :

$$
F_{n}=(1-2 a \Delta t) F_{n-1} .
$$


Inserting the solution

$$
F_{n}=F_{0}(1-2 a \Delta t)^{n}
$$

into the auto-correlation formula of $x$, we get:

$$
C_{x}(t)=(1-2 a \Delta t)^{t / \Delta t} \sim \exp (-2 a t),
$$

where the exponential form is exact in the limit $\Delta t \rightarrow 0$.

The correlation of $r$ can also be expressed in terms of $F_{n}$ :

$$
E\left[r_{t} \cdot r_{0}\right]=E\left[\left(x_{t+\Delta t}-x_{t}\right) \cdot\left(x_{\Delta t}-x_{0}\right)\right]=2 F_{n}-F_{n-1}-F_{n+1} .
$$

Therefore for $n>0$, the auto-correlation takes the form:

$$
C_{r}(t)=\frac{2 F_{n}-F_{n-1}-F_{n+1}}{E\left[\left(x_{\Delta t}-x_{0}\right)^{2}\right]}
$$

Using $E\left[\left(x_{\Delta t}-x_{0}\right)^{2}\right]=4 a \Delta t F_{0}$ and inserting $F_{n}$ into (A12), one proves (19) given in the main text ${ }^{15}$. Eq. (19) can be alternatively expressed by means of the following approximation:

$$
F_{n+1}-2 F_{n}+F_{n-1}=\Delta t^{2} \frac{\partial^{2} F_{n}}{\partial t^{2}}=(a \Delta t)^{2} \exp (-2 a t) .
$$

Plugging eq. (A13) into eq. (A12), we arrive to the same result.

Computation of $C_{r^{2}}$ is slightly more complicated. In order to compute the autocorrelation function of the second moment, we use eq. (29) to substitute for the auto-covariances of $r_{t}^{2}$ :

$$
\begin{aligned}
& E\left[r_{t}^{2} \cdot r_{0}^{2}\right]= \\
& E\left[\left(x_{t+\Delta t}-x_{t}\right)^{2} \cdot\left(x_{\Delta t}-x_{0}\right)^{2}\right]= \\
& E\left[\left(-2 a \Delta t x_{t}+\sqrt{2 b \Delta t\left(1-x_{t}^{2}\right)} \eta_{t}\right)^{2} \cdot\left(-2 a \Delta t x_{0}+\sqrt{2 b \Delta t\left(1-x_{0}^{2}\right)} \eta_{0}\right)^{2}\right]= \\
& 4 b^{2} \Delta t^{2} E\left[1-x_{t}^{2} \eta_{0}^{2}-x_{0}^{2} \eta_{0}^{2}+x_{t}^{2} x_{0}^{2} \eta_{0}^{2}\right]+o\left(\Delta t^{2}\right) .
\end{aligned}
$$

It is obvious, that expression (A14) is identical in leading order $\Delta t^{2}$ with eq. (20) in the main text. It can be easily derived from eq. (14) that $E\left[x_{t}^{2}\right]=\frac{1}{2 \epsilon+1}$. Then, from eq. (29), we can work out the following relation:

$$
E\left[x_{t}^{2} \eta_{0}^{2}\right]=(1-2 b \Delta t(2 \varepsilon+1)) E\left[x_{t-\Delta t}^{2} \eta_{0}^{2}\right]+2 b \Delta t \quad \text { for } t>1
$$

\footnotetext{
${ }^{15}$ For $F_{n+1}$ and $F_{n-1}$ we use the Taylor expansion $e^{x}=1+x+\frac{x^{2}}{2}+o\left(x^{2}\right)$.
} 
Defining $H_{n}=E\left[x_{n \Delta t}^{2} \eta_{0}^{2}\right]$ and $H_{0}=E\left[x_{0}^{2}\right]$, the recursive approach leads to:

$$
H_{n}=\frac{8 a \Delta t E\left[x_{0}^{2}\right]}{(1-2 b \Delta t(2 \varepsilon+1))} \exp (-2 b n \Delta t(2 \varepsilon+1))+E\left[x_{0}^{2}\right]=E\left[x_{0}^{2}\right]+o(\Delta t) .
$$

The infinitesimal term $o(\Delta t)$ in eq. (A16) stands for the contribution to the recursive eq. (A15) of the the term $E\left[x_{1}^{2} \eta_{0}^{2}\right]=(1-2 b \Delta t(2 \varepsilon+3)) E\left[x_{0}^{2}\right]+6 b \Delta t$, which is slightly different from the other terms in the recursive eq. (A15). In order to obtain a closedform solution for $E\left[x_{t}^{2} \cdot x_{0}^{2} \cdot \eta_{0}^{2}\right]$, we again make use of a recursive determination of the auto-covariances. Defining $G_{n}=E\left[x_{n \Delta t}^{2} x_{0}^{2} \eta_{0}^{2}\right]$, we can write:

$$
G_{n+1}=(1-2 b \Delta t(2 \epsilon+1)) G_{n}+2 b \Delta t E\left[x_{0}^{2}\right] \text { for } t>1,
$$

Solving this difference equation, we arrive at the following expression:

$$
G_{n}=(1-2 b \Delta t(2 \epsilon+1))^{n}\left(G_{0}-E\left[x_{0}^{2}\right]^{2}\right)+E\left[x_{0}^{2}\right]^{2}+o(\Delta t),
$$

with $G_{0}=E\left[x_{0}^{4}\right]$. The infinitesimal term takes into account the contribution to the auto-covariance of the term $E\left[x_{\Delta t}^{2} x_{0}^{2} \eta_{0}^{2}\right]$. From (14), we obtain the fourth moment of $x_{0}$ :

$$
G_{0}=E\left[x_{0}^{4}\right]=\frac{3}{(2 \epsilon+3)(2 \epsilon+1)} .
$$

In order to solve for $C_{r^{2}}(t)$, we need some further ingredients:

$$
\begin{aligned}
E\left[r_{0}^{2}\right]=E\left[\left(x_{\Delta t}-x_{0}\right)^{2}\right] & =E\left[\left(-2 a \Delta t x_{0}+\sqrt{2 b \Delta t\left(1-x_{0}^{2}\right)} \eta_{0}\right)^{2}\right] \\
& =2 b \Delta t E\left[\left(1-x_{0}^{2}\right) \eta_{0}^{2}\right]+o(\Delta t) \simeq 2 b \Delta t\left(1-E\left[x_{0}^{2}\right]\right) .
\end{aligned}
$$

We, therefore, get for the numerator of $C_{r^{2}}(t)$ in leading order of $\Delta t$ :

$$
E\left[r_{t+\Delta t}^{2} \cdot r_{\Delta t}^{2}\right]-E\left[r_{\Delta t}^{2}\right]=4 b^{2} \Delta t^{2}(1-2 b \Delta t(2 \epsilon+1))^{t / \Delta t}\left(E\left[x_{0}^{4}\right]-E\left[x_{0}^{2}\right]^{2}\right) .
$$

Finally, in order to pin down the denominator, consider

$$
\begin{aligned}
E\left[r_{0}^{4}\right] & =E\left[\left(-2 a \Delta t x_{0}+\sqrt{2 b \Delta t\left(1-x_{0}^{2}\right)} \eta_{0}\right)^{4}\right] \\
& =E\left[(2 b \Delta t)^{2}\left(1-x_{0}^{2}\right)^{2} \eta_{0}^{4}\right]+o\left(\Delta t^{2}\right) \\
& \simeq(2 b \Delta t)^{2} E\left[\left(1-2 x_{0}^{2}+x_{0}^{4}\right) \eta_{0}^{4}\right] \\
& =3(2 b \Delta t)^{2} \frac{4 \epsilon(\epsilon+1)}{(2 \epsilon+1)(2 \epsilon+3)} .
\end{aligned}
$$

It, then, turns out that:

$$
E\left[r_{0}^{4}\right]-E\left[r_{0}^{2}\right]^{2}=(2 b \Delta t)^{2} \frac{\left(4 \epsilon^{2}+6 \epsilon+3\right) 4 \epsilon}{(2 \epsilon+1)^{2}(2 \epsilon+3)} .
$$

One solves for

$$
C_{r^{2}}(t)=\frac{E\left[r_{t+\Delta t}^{2} \cdot r_{\Delta t}^{2}\right]-E\left[r_{t}^{2}\right]^{2}}{E\left[r_{t}^{4}\right]-E\left[r_{t}^{2}\right]^{2}},
$$

and obtains eq. (21). 


\section{A.4 Mean First Passage Time}

To compute the MFPT $T\left(x_{0} \rightarrow x_{2}\right)$ one integrates the Fokker Planck equation with a reflecting boundary condition at $x=-1$ and an absorbing boundary condition at $x=+1$. Evoking the following general formula (see Gardiner [2003]) for MPFTs, we can try to find a closed-form solution for this quantity as well:

$$
T\left(x_{0} \rightarrow x_{2}\right)=\int_{x_{0}}^{x_{2}} d x(\psi(x))^{-1} \int_{x}^{x_{1}} \frac{2 \psi(y)}{D(y)} d y
$$

with the auxiliary $\psi(x)$ function being defined as:

$$
\ln \psi(x)=\int_{0}^{x} \frac{2 A(y)}{D(y)} d y .
$$

Note first that with drift and diffusion terms $A(x)=-2 a x$ and $D(x)=2 b\left(1-x^{2}\right)$, according to our previous computations $\psi(x)=\left(1-x^{2}\right)^{\varepsilon}$. Taking stock of this result, we obtain:

$$
b T(-1 \rightarrow 1)=\int_{-1}^{1}\left(1-x^{2}\right)^{-\varepsilon}\left(\int_{-1}^{x} \frac{2\left(1-y^{2}\right)^{\varepsilon}}{2 b\left(1-y^{2}\right)} d y\right) d x
$$

Because of the symmetry of $\left(1-y^{2}\right)^{\varepsilon-1}$ we can solve the double integral in a few steps:

$$
b T(-1 \rightarrow 1)=\int_{-1}^{1}\left(1-x^{2}\right)^{-\varepsilon}\left(\int_{-1}^{0}\left(1-y^{2}\right)^{\varepsilon-1} d y-\int_{0}^{-x}\left(1-y^{2}\right)^{\varepsilon-1} d y\right) d x
$$

where the second integral is identically zero since the integrand is an odd function integrated in a symmetric interval. We arrive, then, at:

$$
\begin{aligned}
b T(-1 \rightarrow 1) & =\frac{1}{2} \int_{-1}^{1}\left(1-x^{2}\right)^{-\varepsilon} d x \int_{-1}^{1}\left(1-y^{2}\right)^{\varepsilon-1} d y \\
& =\frac{1}{2} \int_{-1}^{1} 2^{2 \varepsilon-1} \frac{\Gamma(\varepsilon)^{2}}{\Gamma(2 \varepsilon)}\left(1-x^{2}\right)^{-\varepsilon} d x \\
& =\frac{1}{2} \cdot \frac{\Gamma(\varepsilon)^{2} \cdot \Gamma(1-\varepsilon)^{2}}{\Gamma(2 \varepsilon) \cdot \Gamma(2-2 \varepsilon)} .
\end{aligned}
$$

The identity $\Gamma(1-x) \cdot \Gamma(x)=\frac{\pi}{\sin (\pi x)}$ finally leads to:

$$
T(-1 \rightarrow 1)=\frac{1}{b} \frac{\pi}{(1-2 \varepsilon)} \cdot \frac{\cos (\pi \varepsilon)}{\sin (\pi \varepsilon)}
$$




\section{A.5 Convergence to the Gaussian}

We have shown that in the case of local interactions the drift and diffusion terms are given by eq. (11), where $a=0.5 / N_{0}$. Given the N-dependence of $b$, the $1 / N$ correction to the diffusion can not be neglected any longer, and both terms have to be taken into account in deriving the equilibrium distribution. For the number of agents $N$ much higher than the benchmark value $N_{0}$, the equilibrium distribution converges to a Gaussian. Using eq. (A8), we obtain:

$$
\omega_{e}(x) \propto\left(1-x^{2}+\frac{2 a}{\bar{b}}\right)^{\varepsilon_{N}-1},
$$

where $\varepsilon_{N}=\frac{N}{2 \bar{b} N_{0}}$. The previous expression can be rewritten as:

$$
\omega_{e}(x) \propto \exp \left(\left(\varepsilon_{N}-1\right) \ln \left(1-x^{2}+\frac{2 a}{\bar{b}}\right)\right) .
$$

Recalling eq. (13), the equilibrium distribution is well approximated by a Gaussian with mean zero and variance:

$$
\sigma^{2}=\frac{1}{2\left(\frac{N}{2 \bar{b} N_{0}}-1\right)} \approx \frac{\bar{b} N_{0}}{N} .
$$

\section{A.6 Langevin equation}

The basic idea of the Langevin approach is to find an appropriate time interval, which one might call a macro-interval, for which the conditional distribution of the discrete variable $x$ is well approximated by a Gaussian. In the following we sketch a proof of the Langevin equation as an approximation to the discrete process (3) based on some heuristic arguments. Let us start with the identity:

$$
x_{t+\Delta t}=x_{t}+\sum_{i}^{M} \eta_{t+i \Delta t_{0}},
$$

where $\Delta t=M \Delta t_{0}$ and $\eta\left(t+i \Delta t_{0}\right)$ might take the values in the finite set $S \equiv$ $\{0,2 / N,-2 / N\} ; \Delta t_{0}$ is the elementary time interval for a possible change of strategy of one agent. The previous decomposition is based on a separation of the dynamics of $x$ into two different time scales: a micro time scale $\Delta t_{0}$ and a macro time scale $\Delta t$. During the micro interval $\Delta t_{0}$, the variation of $x$ is constrained to the numerable set $S$. If we concentrate on the aggregate variation $\Delta x$ during the macro-time interval $\Delta t$, we sum up over many of these elementary increments, loosing the information on the fine structure of the dynamics of $x$ (i.e. the single movements of the distinguishing individuals). Our aim is exactly to give up this too detailed perspective, 
in favor of a more general macroscopic description of the system. In order to do so, we have to find a proper time scale, long enough to aggregate the tiny details, and not too long to lose relevant information. This perspective is sometimes called a mesoscopic approach, as it is somehow between the micro and macroscopic approach [Ramsey, 1996]. We have, then, to find the proper value for the aggregation level $M$. The random variable $\eta\left(t+(i+1) \Delta t_{0}\right)$ can take just three values with the following probabilities:

$$
\eta_{i+1}=\left\{\begin{array}{cl}
+\frac{1}{N} & p_{i} \Delta t_{0}=\left(1-x_{i}\right)\left(\frac{2 \varepsilon}{N}+1+x_{i}\right) b N^{2} \Delta t_{0} \\
0 & 1-\left(p_{i}+q_{i}\right) \Delta t_{0} \\
-\frac{1}{N} & q_{i} \Delta t_{0}=\left(1+x_{i}\right)\left(\frac{2 \varepsilon}{N}+1-x_{i}\right) b N^{2} \Delta t_{0}
\end{array}\right.
$$

where we explicitly take into account the dependence on the previous value of $x$ (here $x_{i}=x\left(t+i \Delta t_{0}\right)$ and $\left.\eta_{i+1}=\eta\left(t+(i+1) \Delta t_{0}\right)\right)$. For notational convenience, we label the time step by $i$. The mean $\mu_{i+1}$ and variance $\sigma_{i+1}$ of $\eta_{i+1}$ are given by, respectively:

$$
\begin{aligned}
\mu_{i+1} & =\frac{\Delta t_{0}}{N}\left(p_{i}-q_{i}\right), \\
\sigma_{i+1}^{2} & =E\left[\eta_{i+1}^{2}\right]-\mu_{r_{+1}}^{2}=\frac{\Delta t_{0}}{N^{2}}\left(p_{i}+q_{i}\right)+o\left(\frac{\Delta t_{0}}{N^{2}}\right) .
\end{aligned}
$$

Following the Central Limit Theorem (CLT), a sum of $M$ independent random variables, drawn from a common distribution, with mean $\mu$ and finite variance $\sigma^{2}$, converges to a Normal with mean $M \mu$ and variance $M \sigma^{2}$. We can, therefore, approximate the sum in (A17) by a normally distributed random variable. The requirement of a finite variance is certainly fulfilled by the distribution of the variable $\eta$. However, the iid-ness assumption does not strictly hold. The probabilities $p \Delta t_{0}$ and $q \Delta t_{0}$ depend on $x$, which dynamically changes with $\eta$ itself. We have to impose a further condition on $x$ : it should not vary 'too much' during the time interval $\Delta t$, in such a way that it can be treated as a constant. Finally, $M$ and, consequentially, $\Delta t$ should be large enough to assure the convergence towards the Gaussian, but not too large to prevent the approximation of constant $x$.

We approximate the sum in eq. (A17) by a Gaussian distribution with mean $M \mu$ and standard deviation $\sqrt{M} \sigma$. Therefore we end up with:

$$
x_{t+\Delta t}=x_{t}-2 a x_{t} M \Delta t_{0}+\sqrt{2\left(1-x_{t}^{2}\right) M b \Delta t_{0}} \cdot \eta_{t},
$$

where $\eta_{t}$ is a Normally distributed random variable. The inequality $M b \Delta t_{0} \ll 1$ should be satisfied in order to guarantee small variations of the variable $x$ and, therefore, to preserve the approximation of a constant $x$ during the interval $\Delta t$. 
Recalling the inequality (22), we come up with the following scaling relation between the number of micro steps $M$ and the number of agents $N$ :

$$
M=k \frac{N^{2}}{2},
$$

with an arbitrary small number $k$. Finally, the Langevin approximation for the stochastic process eq. (A17) is given by:

$$
\Delta x=-2 a \Delta t x_{t}+\sqrt{2 b \Delta t\left(1-x_{t}^{2}\right)} \cdot \eta_{t},
$$

where $\Delta t=M \Delta t_{0}$ The limit of such an approximation is related to the assumption of the CLT, therefore to the goodness of the Gaussian approximation for the sum in eq. (A17). Near the edges, $x=-1$ or $x=1$, the variable $x$ cannot be considered to be constant, and the assumption of identically distributed variables does not hold any longer ${ }^{16}$. Additionally, eq. (A23) does not incorporate neither the conditions of boundedness of $x$ to the compact interval $[-1,1]$, nor the natural boundary conditions of the eqs. (1) and (3). The boundary conditions, then, should be put in the equation (A23) 'by hand'. They are given by:

$$
\begin{aligned}
\text { if } \quad x(t)>1 \quad \text { then } \quad & \frac{x(t+\Delta t)+x(t)}{2}=1, \\
\text { if } \quad x(t)<-1 \quad \text { then } \quad \frac{x(t+\Delta t)+x(t)}{2} & =-1 .
\end{aligned}
$$

A glance to eqs. (A24) and (A25) shows that they are equivalent to a reflection around the edges of the domain of $x, x=1$ and $x=-1$, respectively. ${ }^{17}$

\footnotetext{
${ }^{16}$ Note that also the FPE is not a valid description of the system near the borders.

${ }^{17}$ Alternative boundary conditions are also possible. For example, the boundary conditions can also be implemented as follows:

$$
\begin{aligned}
\text { if } \quad x(t)>1 \quad \text { then } \quad x(t+\Delta t)=1, \\
\text { if } \quad x(t)<-1 \quad \text { then } \quad x(t+\Delta t)=-1 .
\end{aligned}
$$
}




\section{List of other working papers:}

\section{5}

1. Shaun Bond and Soosung Hwang, Smoothing, Nonsynchronous Appraisal and CrossSectional Aggreagation in Real Estate Price Indices, WP05-17

2. Mark Salmon, Gordon Gemmill and Soosung Hwang, Performance Measurement with Loss Aversion, WP05-16

3. Philippe Curty and Matteo Marsili, Phase coexistence in a forecasting game, WP05-15

4. Matthew Hurd, Mark Salmon and Christoph Schleicher, Using Copulas to Construct Bivariate Foreign Exchange Distributions with an Application to the Sterling Exchange Rate Index (Revised), WP05-14

5. Lucio Sarno, Daniel Thornton and Giorgio Valente, The Empirical Failure of the Expectations Hypothesis of the Term Structure of Bond Yields, WP05-13

6. Lucio Sarno, Ashoka Mody and Mark Taylor, A Cross-Country Financial Accelorator: Evidence from North America and Europe, WP05-12

7. Lucio Sarno, Towards a Solution to the Puzzles in Exchange Rate Economics: Where Do We Stand?, WP05-11

8. James Hodder and Jens Carsten Jackwerth, Incentive Contracts and Hedge Fund Management, WP05-10

9. James Hodder and Jens Carsten Jackwerth, Employee Stock Options: Much More Valuable Than You Thought, WP05-09

10. Gordon Gemmill, Soosung Hwang and Mark Salmon, Performance Measurement with Loss Aversion, WP05-08

11. George Constantinides, Jens Carsten Jackwerth and Stylianos Perrakis, Mispricing of S\&P 500 Index Options, WP05-07

12. Elisa Luciano and Wim Schoutens, A Multivariate Jump-Driven Financial Asset Model, WP0506

13. Cees Diks and Florian Wagener, Equivalence and bifurcations of finite order stochastic processes, WP05-05

14. Devraj Basu and Alexander Stremme, CAY Revisited: Can Optimal Scaling Resurrect the (C)CAPM?, WP05-04

15. Ginwestra Bianconi and Matteo Marsili, Emergence of large cliques in random scale-free networks, WP05-03

16. Simone Alfarano, Thomas Lux and Friedrich Wagner, Time-Variation of Higher Moments in a Financial Market with Heterogeneous Agents: An Analytical Approach, WP05-02

17. Abhay Abhayankar, Devraj Basu and Alexander Stremme, Portfolio Efficiency and Discount Factor Bounds with Conditioning Information: A Unified Approach, WP05-01

\section{4}

1. Xiaohong Chen, Yanqin Fan and Andrew Patton, Simple Tests for Models of Dependence Between Multiple Financial Time Series, with Applications to U.S. Equity Returns and Exchange Rates, WP04-19

2. Valentina Corradi and Walter Distaso, Testing for One-Factor Models versus Stochastic Volatility Models, WP04-18

3. Valentina Corradi and Walter Distaso, Estimating and Testing Sochastic Volatility Models using Realized Measures, WP04-17

4. Valentina Corradi and Norman Swanson, Predictive Density Accuracy Tests, WP04-16

5. Roel Oomen, Properties of Bias Corrected Realized Variance Under Alternative Sampling Schemes, WP04-15

6. Roel Oomen, Properties of Realized Variance for a Pure Jump Process: Calendar Time Sampling versus Business Time Sampling, WP04-14 
7. Richard Clarida, Lucio Sarno, Mark Taylor and Giorgio Valente, The Role of Asymmetries and Regime Shifts in the Term Structure of Interest Rates, WP04-13

8. Lucio Sarno, Daniel Thornton and Giorgio Valente, Federal Funds Rate Prediction, WP04-12

9. Lucio Sarno and Giorgio Valente, Modeling and Forecasting Stock Returns: Exploiting the Futures Market, Regime Shifts and International Spillovers, WP04-11

10. Lucio Sarno and Giorgio Valente, Empirical Exchange Rate Models and Currency Risk: Some Evidence from Density Forecasts, WP04-10

11. Ilias Tsiakas, Periodic Stochastic Volatility and Fat Tails, WP04-09

12. Ilias Tsiakas, Is Seasonal Heteroscedasticity Real? An International Perspective, WP04-08

13. Damin Challet, Andrea De Martino, Matteo Marsili and Isaac Castillo, Minority games with finite score memory, WP04-07

14. Basel Awartani, Valentina Corradi and Walter Distaso, Testing and Modelling Market Microstructure Effects with an Application to the Dow Jones Industrial Average, WP04-06

15. Andrew Patton and Allan Timmermann, Properties of Optimal Forecasts under Asymmetric Loss and Nonlinearity, WP04-05

16. Andrew Patton, Modelling Asymmetric Exchange Rate Dependence, WP04-04

17. Alessio Sancetta, Decoupling and Convergence to Independence with Applications to Functional Limit Theorems, WP04-03

18. Alessio Sancetta, Copula Based Monte Carlo Integration in Financial Problems, WP04-02

19. Abhay Abhayankar, Lucio Sarno and Giorgio Valente, Exchange Rates and Fundamentals: Evidence on the Economic Value of Predictability, WP04-01

\section{2}

1. Paolo Zaffaroni, Gaussian inference on Certain Long-Range Dependent Volatility Models, WP02-12

2. Paolo Zaffaroni, Aggregation and Memory of Models of Changing Volatility, WP02-11

3. Jerry Coakley, Ana-Maria Fuertes and Andrew Wood, Reinterpreting the Real Exchange Rate - Yield Diffential Nexus, WP02-10

4. Gordon Gemmill and Dylan Thomas, Noise Training, Costly Arbitrage and Asset Prices: evidence from closed-end funds, WP02-09

5. Gordon Gemmill, Testing Merton's Model for Credit Spreads on Zero-Coupon Bonds, WP0208

6. George Christodoulakis and Steve Satchell, On th Evolution of Global Style Factors in the MSCI Universe of Assets, WP02-07

7. George Christodoulakis, Sharp Style Analysis in the MSCI Sector Portfolios: A Monte Caro Integration Approach, WP02-06

8. George Christodoulakis, Generating Composite Volatility Forecasts with Random Factor Betas, WP02-05

9. Claudia Riveiro and Nick Webber, Valuing Path Dependent Options in the Variance-Gamma Model by Monte Carlo with a Gamma Bridge, WP02-04

10. Christian Pedersen and Soosung Hwang, On Empirical Risk Measurement with Asymmetric Returns Data, WP02-03

11. Roy Batchelor and Ismail Orgakcioglu, Event-related GARCH: the impact of stock dividends in Turkey, WP02-02

12. George Albanis and Roy Batchelor, Combining Heterogeneous Classifiers for Stock Selection, WP02-01

\section{1}

1. Soosung Hwang and Steve Satchell, GARCH Model with Cross-sectional Volatility; GARCHX Models, WP01-16

2. Soosung Hwang and Steve Satchell, Tracking Error: Ex-Ante versus Ex-Post Measures, WP01-15

3. Soosung Hwang and Steve Satchell, The Asset Allocation Decision in a Loss Aversion World, WP01-14

4. Soosung Hwang and Mark Salmon, An Analysis of Performance Measures Using Copulae, WP01-13

5. Soosung Hwang and Mark Salmon, A New Measure of Herding and Empirical Evidence, WP01-12 
6. Richard Lewin and Steve Satchell, The Derivation of New Model of Equity Duration, WP0111

7. Massimiliano Marcellino and Mark Salmon, Robust Decision Theory and the Lucas Critique, WP01-10

8. Jerry Coakley, Ana-Maria Fuertes and Maria-Teresa Perez, Numerical Issues in Threshold Autoregressive Modelling of Time Series, WP01-09

9. Jerry Coakley, Ana-Maria Fuertes and Ron Smith, Small Sample Properties of Panel Timeseries Estimators with I(1) Errors, WP01-08

10. Jerry Coakley and Ana-Maria Fuertes, The Felsdtein-Horioka Puzzle is Not as Bad as You Think, WP01-07

11. Jerry Coakley and Ana-Maria Fuertes, Rethinking the Forward Premium Puzzle in a Nonlinear Framework, WP01-06

12. George Christodoulakis, Co-Volatility and Correlation Clustering: A Multivariate Correlated ARCH Framework, WP01-05

13. Frank Critchley, Paul Marriott and Mark Salmon, On Preferred Point Geometry in Statistics, WP01-04

14. Eric Bouyé and Nicolas Gaussel and Mark Salmon, Investigating Dynamic Dependence Using Copulae, WP01-03

15. Eric Bouyé, Multivariate Extremes at Work for Portfolio Risk Measurement, WP01-02

16. Erick Bouyé, Vado Durrleman, Ashkan Nikeghbali, Gael Riboulet and Thierry Roncalli, Copulas: an Open Field for Risk Management, WP01-01

\section{0}

1. Soosung Hwang and Steve Satchell, Valuing Information Using Utility Functions, WP00-06

2. Soosung Hwang, Properties of Cross-sectional Volatility, WP00-05

3. Soosung Hwang and Steve Satchell, Calculating the Miss-specification in Beta from Using a Proxy for the Market Portfolio, WP00-04

4. Laun Middleton and Stephen Satchell, Deriving the APT when the Number of Factors is Unknown, WP00-03

5. George A. Christodoulakis and Steve Satchell, Evolving Systems of Financial Returns: AutoRegressive Conditional Beta, WP00-02

6. Christian S. Pedersen and Stephen Satchell, Evaluating the Performance of Nearest Neighbour Algorithms when Forecasting US Industry Returns, WP00-01

\section{9}

1. Yin-Wong Cheung, Menzie Chinn and Ian Marsh, How do UK-Based Foreign Exchange Dealers Think Their Market Operates?, WP99-21

2. Soosung Hwang, John Knight and Stephen Satchell, Forecasting Volatility using LINEX Loss Functions, WP99-20

3. Soosung Hwang and Steve Satchell, Improved Testing for the Efficiency of Asset Pricing Theories in Linear Factor Models, WP99-19

4. Soosung Hwang and Stephen Satchell, The Disappearance of Style in the US Equity Market, WP99-18

5. Soosung Hwang and Stephen Satchell, Modelling Emerging Market Risk Premia Using Higher Moments, WP99-17

6. Soosung Hwang and Stephen Satchell, Market Risk and the Concept of Fundamental Volatility: Measuring Volatility Across Asset and Derivative Markets and Testing for the Impact of Derivatives Markets on Financial Markets, WP99-16

7. Soosung Hwang, The Effects of Systematic Sampling and Temporal Aggregation on Discrete Time Long Memory Processes and their Finite Sample Properties, WP99-15

8. Ronald MacDonald and Ian Marsh, Currency Spillovers and Tri-Polarity: a Simultaneous Model of the US Dollar, German Mark and Japanese Yen, WP99-14

9. Robert Hillman, Forecasting Inflation with a Non-linear Output Gap Model, WP99-13

10. Robert Hillman and Mark Salmon, From Market Micro-structure to Macro Fundamentals: is there Predictability in the Dollar-Deutsche Mark Exchange Rate?, WP99-12

11. Renzo Avesani, Giampiero Gallo and Mark Salmon, On the Evolution of Credibility and Flexible Exchange Rate Target Zones, WP99-11

12. Paul Marriott and Mark Salmon, An Introduction to Differential Geometry in Econometrics, WP99-10 
13. Mark Dixon, Anthony Ledford and Paul Marriott, Finite Sample Inference for Extreme Value Distributions, WP99-09

14. Ian Marsh and David Power, A Panel-Based Investigation into the Relationship Between Stock Prices and Dividends, WP99-08

15. Ian Marsh, An Analysis of the Performance of European Foreign Exchange Forecasters, WP99-07

16. Frank Critchley, Paul Marriott and Mark Salmon, An Elementary Account of Amari's Expected Geometry, WP99-06

17. Demos Tambakis and Anne-Sophie Van Royen, Bootstrap Predictability of Daily Exchange Rates in ARMA Models, WP99-05

18. Christopher Neely and Paul Weller, Technical Analysis and Central Bank Intervention, WP9904

19. Christopher Neely and Paul Weller, Predictability in International Asset Returns: A Reexamination, WP99-03

20. Christopher Neely and Paul Weller, Intraday Technical Trading in the Foreign Exchange Market, WP99-02

21. Anthony Hall, Soosung Hwang and Stephen Satchell, Using Bayesian Variable Selection Methods to Choose Style Factors in Global Stock Return Models, WP99-01

1998

1. Soosung Hwang and Stephen Satchell, Implied Volatility Forecasting: A Compaison of Different Procedures Including Fractionally Integrated Models with Applications to UK Equity Options, WP98-05

2. Roy Batchelor and David Peel, Rationality Testing under Asymmetric Loss, WP98-04

3. Roy Batchelor, Forecasting T-Bill Yields: Accuracy versus Profitability, WP98-03

4. Adam Kurpiel and Thierry Roncalli, Option Hedging with Stochastic Volatility, WP98-02

5. Adam Kurpiel and Thierry Roncalli, Hopscotch Methods for Two State Financial Models, WP98-01 